\title{
PERSPECTIVES ON SCISSORS CONGRUENCE
}

\author{
INNA ZAKHAREVICH
}

\begin{abstract}
In this paper we give a short introduction to the different theories of scissors congruence. We begin with classical scissors congruence, which considers equivalence classes of polyhedra under dissection. We then move to multi-dimensional scissors congruence along the lines of McMullen's polytope algebra and then to the Grothendieck ring of varieties. Tying our discussion together is the question of whether algebraic invariants are sufficient to distinguish scissors congruence classes.
\end{abstract}

\section{INTRODUCTION}

Scissors congruence is the study of whether two objects are the same under decomposition. When solving problems it is often necessary to decompose the problem into smaller pieces, solve each piece separately, and then assemble the pieces into a solution for the original problem. The study of the indeterminacy of this - the question of how many different objects can be assembled from the given piecesis the fundamental question of scissors congruence. Classically, this question has been asked about polyhedra in Euclidean geometry, but similar questions crop up in algebraic $K$-theory, surgery on manifolds, and motivic integration.

Different notions of congruence arise from different choices of allowable motions. For example, consider the question of general scissors congruence in $\mathbb{R}^{3}$. Two subsets $S$ and $T$ of $\mathbb{R}^{3}$ are scissors congruent if it is possible to write

$$
S=\biguplus_{i=1}^{n} S_{i} \quad \text { and } \quad T=\biguplus_{i=1}^{n} T_{i}
$$

and there exist isometries $\varphi_{i}$ of $\mathbb{R}^{3}$ such that $\varphi_{i}\left(S_{i}\right)=T_{i}$. Here, $\biguplus$ denotes the assumption that $S_{i} \cap S_{j}=\emptyset$ when $i \neq j$; we use the $\biguplus$ notation to emphasize that the union is taking place in $\mathbb{R}^{3}$. When are two sets scissors congruent? The Banach-Tarski paradox states that any two bounded sets with nonempty interiors inside $\mathbb{R}^{3}$ are scissors congruent; thus no "geometric" notion of scissors congruence invariants is possible. To avoid such paradoxes, scissors congruence is generally studied with strict restrictions on the kinds of sets allowed. This paper focuses on polyhedral and algebraic scissors congruence, but other contexts are possible. For example, for a discussion of scissors congruence in the context of tame topology, see Dri98.

One major tension in the analysis of scissors congruence invariants is the difference between geometric analysis and algebraic analysis. In the geometric analysis, objects under consideration are studied directly, and explicit scissors congruences are constructed between them. The advantage of this approach is that no geometric

Received by the editors October 2, 2015.

2010 Mathematics Subject Classification. 52B45. 
information is lost; the disadvantage is that it is often prohibitively difficult. In the algebraic analysis, the object under study is a group representing the universal algebraic scissors congruence invariant - in other words the universal abelian group $G$ for which there exists a function

\section{$f:\{$ objects under study $\} \longrightarrow G$}

such that if $S$ and $T$ are scissors congruent, then $f(S)=f(T)$. This group can then be studied using algebraic tools such as group homology. The advantage of this approach is that significant progress can often be made. The disadvantage is that it can lose significant geometric information: it may be the case that two objects always have the same algebraic invariants but are not scissors congruent.

This tension will be our guide through this paper. In any scissors congruence context, we may ask whether the following Property $2^{1}$ holds:

Property Z: If two objects $S$ and $T$ are indistinguishable by algebraic scissors congruence invariants, then they are scissors congruent.

The first three sections of the paper examine whether Property $\mathrm{Z}$ holds in different scissors congruence contexts, from classical scissors congruence (Section 1) to multi-dimensional scissors congruence (Section 2) to the Grothendieck ring of varieties (Section 3). Each of these topics is beautiful and interesting in its own right, and we cannot hope to do them justice in a single paper. For the interested reader we recommend the following: For an introduction to classical scissors congruence we recommend Cartier's Bourbaki seminar paper Car86] and Sah's book [Sah79]. For an introduction to multi-dimensional scissors congruence we recommend McMullen's work [McM89]. For an overview of the theory of the Grothendieck ring of varieties we recommend Nicasise and Sebag's article [NS11. However, it is difficult to resist the temptation to discuss some of the topics that come up in this subject, so each section of this paper ends with a short discussion of other topics that come up in the study of scissors congruence.

The last section of this paper shows how to unify all of these contexts into a single framework which is also well suited for analyzing the difference between the geometric and algebraic approaches. The framework is based on the machinery of algebraic $K$-theory. In general, algebraic $K$-theory asks the question of what information is lost when group-completing, and whether it is possible to recover some of that lost information. This construction generalizes to the context of scissors congruence.

\section{Classical scissors Congruence}

Consider any two three-dimensional polyhedra $P$ and $Q$ in three-dimensional Euclidean space. The fundamental question of scissors congruence is whether it is possible to cut $P$ into finitely many polyhedral pieces and to rearrange the pieces to form $Q$. Such a procedure is a scissors-isomorphism from $P$ to $Q$, written $P \rightsquigarrow Q$. If there exists a scissors-isomorphism from $P$ to $Q$ then $P$ and $Q$ are scissors congruent, written $P \simeq Q$. The first and oldest question about scissors congruence is identifying the scissors-isomorphism invariants. Volume is one, but are there others?

\footnotetext{
${ }^{1}$ Named after Zylev's theorem (see page 273) which establishes it in the case of classical scissors congruence.
} 
For a simpler problem, consider the two-dimensional case where $P$ and $Q$ are polygons. Clearly area is a scissors-isomorphism invariant. To show that there are no other invariants, it suffices to show that there exists a scissors congruence between any two polygons of the same area. To make the problem simpler, notice that scissors-isomorphisms can be composed as illustrated in the following picture. The two scissors-isomorphisms
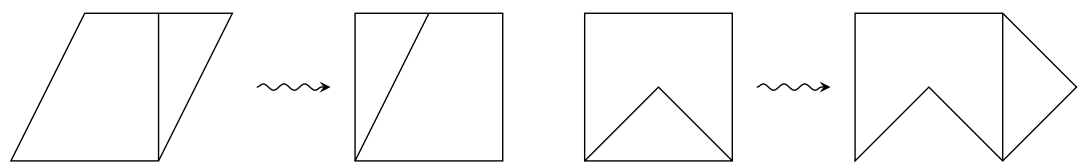

compose to

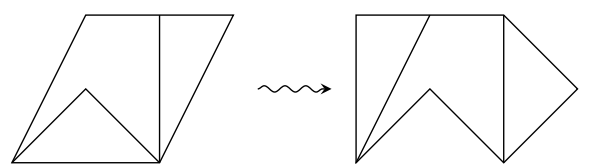

In addition, if there is a scissors-isomorphism $P \rightsquigarrow Q$, then there is also a scissorsisomorphism $Q \rightsquigarrow P$. Thus, scissors congruence is an equivalence relation.

To show that area is the only scissors congruence invariant, it suffices to show that any polygon $P$ has a scissors-isomorphism to an area $(P) \times 1$ rectangle. In fact, since any polygon can be triangulated, it suffices to show this for a triangle. The key steps are transforming a triangle into a rectangle

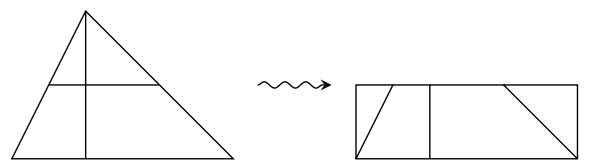

and transforming an $a \times b$ rectangle into an $a b \times 1$ rectangle

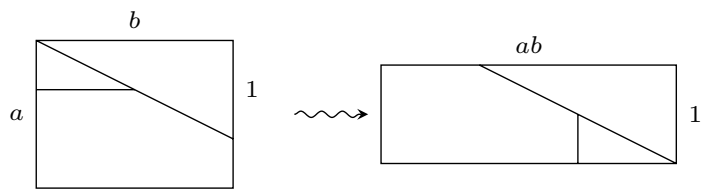

This second construction only works when $2 \geq a \geq 1$, but all other cases can be reduced to this one by either cutting side $a$ into enough pieces for this to hold and doing each piece separately, or by first rearranging it into an $n a \times \frac{b}{n}$ rectangle for a sufficiently large $n$.

Let us turn back to three dimensions. It turns out that there is a second scissors congruence invariant, called the Dehn invariant, constructed by Dehn in 1901. This invariant assigns to each polyhedron an element of $\mathbb{R} \otimes \mathbb{R} / \mathbb{Z}$ by

$$
P \longmapsto \sum_{\text {edges } e} \operatorname{length}(e) \otimes(\theta(e) / \pi),
$$

where $\theta(e)$ is the dihedral angle at the edge $e$, defined as the angle subtended by the two faces of $P$ meeting at $e$ in the plane orthogonal to $e$ through the midpoint of $e$.

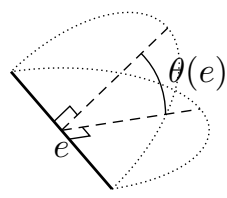


Here, the tensor product is taken with $\mathbb{R}$ and $\mathbb{R} / \mathbb{Z}$ considered as $\mathbb{Q}$-vector spaces. It is elementary to check that this is a well-defined scissors congruence invariant and that the Dehn invariant of a cube is zero; it is a somewhat harder exercise in trigonometry to check that the Dehn invariant of a regular tetrahedron is nonzero. However, once these two facts are established, it immediately follows that volume is not the only scissors congruence invariant: a cube and a tetrahedron with the same volume have different Dehn invariants and, therefore, cannot be scissors congruent.

At this point it is entirely possible that there are still more scissors congruence invariants in three dimensions. Fortunately, in 1965, it was proved by Sydler Syd65] that if two polyhedra have the same volume and Dehn invariant, then they are scissors congruent. This gives a complete description of scissors congruence classes in three dimensions. In four dimensions (with four-dimensional polyhedra) the answer to this question was given by Jessen [Jes68, who managed to simplify Sydler's proof and to show that it extends directly to $n=4$. In this context the Dehn invariant measures angles between three-dimensional faces and takes each two-dimensional face to its area tensored with the angle measure.

Open Question 1. It is possible to generalize the Dehn invariant to higher dimensions (see [Sah79, Section 6.3])? In dimension $n>4$, does the Dehn invariant and the $n$-dimensional volume separate polytopes? In other words, if two $n$-polytopes have the same volume and generalized Dehn invariant, are they scissors congruent?

This problem can also be generalized to other geometries by considering polytopes in three-dimensional spherical and hyperbolic space. As these spaces have more complicated geometry than Euclidean space, it is preferable to switch from the geometric to the algebraic analysis. Toward this end, we define, following Dupont and Sah [Sah79,DS82,Dup01, the scissors congruence groups of a space.

Definition 1.1. Let $X=E^{n}, S^{n}$, or $\mathcal{H}^{n}$ be the $n$-dimensional Euclidean, spherical, or hyperbolic space. Let $G$ be a subgroup of the isometries of $X$. Define

$$
\mathbb{P}(X, G)=\mathbb{Z}\{\text { polytopes in } X\} / \begin{array}{ll}
{[P \cup Q]=[P]+[Q]} & \text { if meas }(P \cap Q)=0, \\
{[g \cdot P]=[P]} & \text { for all } g \in G .
\end{array}
$$

Here, a polytope is a finite union of simplices, and a simplex is the convex hull of $n+1$ points in general position; when $X=S^{n}$, a simplex must be contained in an open hemisphere so that the convex hull is well defined. For conciseness, define $\mathbb{P}(X)$ to be $\mathbb{P}(X, G)$.

In general, write $P \sim Q$ if $[P]=[Q]$ in $\mathbb{P}(X)$. Note that $P \sim Q$ exactly when all algebraic scissors congruence invariants agree on $P$ and $Q$. It is direct from the definition to check that

$$
\mathbb{P}\left(E^{0}\right)=\mathbb{P}\left(S^{0}\right)=\mathbb{P}\left(\mathcal{H}^{0}\right)=\mathbb{Z} \quad \text { and } \quad \mathbb{P}\left(E^{1}\right)=\mathbb{P}\left(S^{1}\right)=\mathbb{P}\left(\mathcal{H}^{1}\right)=\mathbb{R} .
$$

The above discussion of the two-dimensional Euclidean case shows that $\mathbb{P}\left(E^{2}\right)=$ $\mathbb{R}$. We omit the discussion of $\mathbb{P}\left(\mathcal{H}^{2}\right)$ and refer the interested reader to Sah79, Chapter 8] or Dup01, Chapter 8]. To analyze $\mathbb{P}\left(S^{2}\right)$, we observe that there is a homomorphism $\mathbb{P}\left(S^{2}\right) \rightarrow \mathbb{R}$ given by mapping any polytope to its area (normalized so that area $\left.\left(S^{2}\right)=1\right)$; we will show that this is an isomorphism.

Observe that there is a homomorphism $\mathbb{P}\left(S^{1}\right) \rightarrow \mathbb{P}\left(S^{2}\right)$ given by choosing an embedding $S^{1} \rightarrow S^{2}$ as an equator and mapping any circular arc to the triangle which has that arc as an edge and the north pole as the third vertex. The composition 
$\mathbb{P}\left(S^{1}\right) \rightarrow \mathbb{P}\left(S^{2}\right) \rightarrow \mathbb{R}$ is length. Thus, to check that $\mathbb{P}\left(S^{2}\right)=\mathbb{R}$, it suffices to check that the homomorphism $\mathbb{P}\left(S^{1}\right) \rightarrow \mathbb{P}\left(S^{2}\right)$ is surjective, or, in other words, that its cokernel $G$ is trivial. For any point $A \in S^{2}$, write $\bar{A}$ for the point diametrically opposite. Given any spherical triangle $A B C$, the equality $[A B C]+[\bar{A} B C]=0$ holds in $G$, as the following picture illustrates.

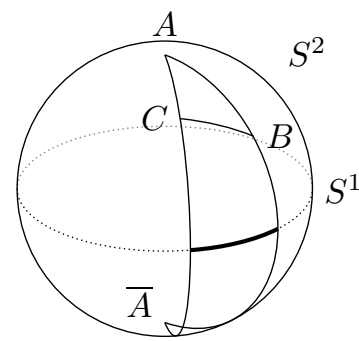

Now consider the following sum in $G$ :

$\alpha=([A B C]+[\bar{A} B C])+([\overline{A B} C]+[\overline{A B C}])=[A B C]+([\bar{A} B C]+[\overline{A B} C])+[\overline{A B C}]$.

By grouping $\alpha$ in the first way, it follows that $\alpha=0$ in $G$. On the other hand, by grouping in the second way it follows that $\alpha=2[A B C]$ in $G$, since $[A B C]=[\overline{A B C}]$. However, $\mathbb{P}\left(S^{2}\right)$ is 2-divisible, since a triangle can be divided into three pairs of congruent triangles by drawing the three angle bisectors; thus $[A B C]=0$ in $G$, and $G$ is trivial, as desired.

This discussion does not quite prove that the only scissors congruence invariant of spherical polygons is area, since it is possible that there exist polygons $P$ and $Q$ such that $P \sim Q$ but $P \nsucceq Q$. In other words, Property $\mathrm{Z}$ has not yet been established.

Theorem 1.2 (Zylev, Sah79, Section 1.3]). For $X=E^{n}, S^{n}$, or $\mathcal{H}^{n}$, if $P \sim Q$, then $P \simeq Q$. In other words, Property $Z$ holds in the case of classical scissors congruence in $E^{n}, S^{n}$, or $\mathcal{H}^{n}$.

In order to illustrate why this theorem is not obvious, consider the case when $X=E^{1}$. Although the theorem can be proved by constructing the length and showing that any two segments with the same length are scissors congruent, this proof does not easily generalize to other geometries and dimensions. In order to prove this theorem in such a way that the proof will generalize to other dimensions and geometries it must be shown that for any segments $P, Q, R$, and $S$ such that $R \simeq S$ and $P \uplus R \simeq Q \uplus S$, it is also the case that $P \simeq Q$; in particular, this must be possible starting with any pair of scissors congruences from $R$ to $S$ and $P \uplus R$ to $Q \uplus S$. Consider segments $P=Q=[0,1]$ and $R=S=[1,2]$. The scissors congruence from $R$ to $S$ is just the trivial one. The scissors congruence from $P \uplus R$ to $Q \uplus S$ writes

$P \uplus R=[0,2]=[0, \sqrt{2}] \uplus[\sqrt{2}, 2] \quad$ and $\quad Q \uplus S=[0,2]=[0,2-\sqrt{2}] \uplus[2-\sqrt{2}, 2]$.

The isometries swap the two segments using translations. In this case there is no direct way of decomposing these segments further to produce a scissors congruence from $P$ to $Q$; something more clever needs to be done.

We prove a much more general theorem, which only relies on the combinatorics of scissors congruence and not on any ambient geometry. First, a couple of definitions:

Property V: If $P^{\prime} \subseteq P$ and $P^{\prime} \simeq P$, then $P^{\prime}=P$. 
Larger than twice: $P$ is said to be larger than twice $Q$ if for any subpolytopes $P^{\prime} \subseteq P$ and $Q^{\prime} \subseteq Q$ such that $P^{\prime} \simeq Q$ there exists a subpolytope $P^{\prime \prime} \subseteq P$ such that $P^{\prime \prime} \cap P^{\prime}=\emptyset$ and $P^{\prime \prime} \simeq Q$.

Property A: For any $P$ and $Q$ there exists a decomposition of $Q=\biguplus_{i=1}^{n} Q_{i}$ such that $P$ is larger than twice $Q_{i}$ for each $i=1, \ldots, n$.

Sah calls Property V the "abstract volume" condition, as it is analogous to there being a volume function on polytopes. It is necessary for Property $\mathrm{Z}$ to hold, as any counterexample to it is also a counterexample to Zylev: if $P^{\prime} \subsetneq P$ is a counterexample, then $\emptyset \cup P \simeq \overline{P \backslash P^{\prime}} \cup P^{\prime}$ and $P \simeq P^{\prime}$ but $\emptyset \neq \overline{P \backslash P^{\prime}}$. Here, the bar notation indicates set-theoretic closure. Property A is an "archimedeanness" condition: it says that anything can be cut up into small enough pieces. This is not necessary for Property $\mathrm{Z}$ to hold, since for example Property $\mathrm{Z}$ holds for segments in the affine line over an ordered non-archimedean field, but the proof no longer works. It can also be thought of as a uniformity condition: if we are considering polytopes of different dimensions, for example, then polytopes with larger dimensions cannot fit into polytopes with smaller dimensions, no matter how much they are cut. Property A also fails when the isometry group of $X$ is not transitive. For example, if we let $X=\overline{\mathcal{H}}^{2}$, the hyperbolic plane with ideal vertices, then in the following picture $A B X A^{\prime} \sim A^{\prime} B^{\prime} \infty$ but $A B X A^{\prime} \nsucceq A^{\prime} B^{\prime} \infty$ as $A B X A^{\prime}$ has no infinite vertices.

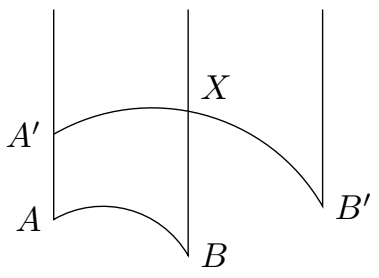

However, in the classical geometries Properties A and V hold, and the following theorem implies Zylev's theorem.

Theorem 1.3 ([Sah79, Section 1.3]). Properties $A$ and $V$ imply Property $Z$.

Remark. We deliberately leave the context of Properties A, V, and Z vague; it is possible to define these properties in much greater generality, and we wish to allude to that generality here. For more detail, see [Sah79, Section 1.3].

Proof. Suppose that $P \sim Q$. This implies that there exist polytopes $R$ and $S$ and scissors-isomorphisms $f: P \uplus R \simeq Q \uplus S$ and $g: R \simeq S$.

First, consider a special case which will contain all of the ideas of the general solution. Suppose in addition that there exists an isometry $\varphi: R \rightsquigarrow S$, and that $P$ is larger than twice $S$. Now suppose that $f$ consists of the following information: four decompositions

$$
P=\biguplus_{i=1}^{m} X_{i}, \quad R=\biguplus_{i=m+1}^{n} X_{i}, \quad Q=\biguplus_{j=1}^{\ell} Y_{j}, \quad S=\biguplus_{j=\ell+1}^{n} Y_{j},
$$

a permutation $\sigma \in \Sigma_{n}$, and isometries $f_{i}: X_{i} \rightarrow Y_{\sigma(i)}$. Let

$$
R^{\prime}=\biguplus_{j=\ell+1}^{n} X_{\sigma^{-1}(j)}
$$


be the preimage of $S$ under $f$; in particular, $f$ restricts to a scissors-isomorphism $R^{\prime} \rightsquigarrow S$. There are three possible cases:

CAsE 1: $\sigma\{m+1, \ldots, n\} \subseteq\{\ell+1, \ldots, n\}$. Then the only pieces that hit $S$ come from $R$. By Property V it follows that $R^{\prime}=R$. Thus $m=\ell$ and $\sigma\{1, \ldots, m\}=\{1, \ldots, \ell\}$; in particular, $f$ restricts to a scissors-isomorphism $P \rightsquigarrow Q$.

CASE 2: $\sigma^{-1}\{\ell+1, \ldots, n\} \subseteq\{1, \ldots, m\}$. Then all pieces that hit $S$ come from $P$. Let $g: P \uplus R \rightsquigarrow P \uplus R$ be the scissors-automorphism which swaps $R^{\prime}$ and $R$. More formally, write

$$
P \uplus R=\overline{P \backslash R^{\prime}} \uplus \biguplus_{j=\ell+1}^{n} X_{\sigma^{-1}(j)} \uplus \biguplus_{j=\ell+1}^{n} \varphi^{-1} Y_{j},
$$

and for each $j=\ell+1, \ldots, n$ swap $X_{\sigma^{-1}(j)}$ and $\varphi^{-1} Y_{j}$ using the isometry $g_{j}=$ $\varphi \circ f_{\sigma^{-1}(j)}$ and its inverse. Then the scissors-isomorphism $f \circ g$ reduces the problem to Case 1.

CASE 3: The general case. Write $R^{\prime \prime}=P \cap R^{\prime}$, so that $R^{\prime \prime}$ consists of all pieces of $S$ that come from $P$. Then $R^{\prime \prime}$ is scissors-congruent to a subpolytope of $S$. So by Property A there exists an $S^{\prime} \subseteq P$ such that $S^{\prime} \cap R^{\prime \prime}=\emptyset$ and such that there exists a scissors-isomorphism $g: S^{\prime} \rightsquigarrow S$. Let $\varphi^{-1} g$ be the scissors-isomorphism $S^{\prime} \rightsquigarrow R$ given by composing each isometry in $g$ with $\varphi^{-1}$, and let $h: P \uplus R \rightsquigarrow P \uplus R$ be the scissors-automorphism that swaps $S^{\prime}$ and $R$ (analogously to the way it was done in Case 2). The scissors-isomorphism $f \circ h$ reduces the problem to Case 2 .

All together, these show that in the special case under consideration it is possible to construct a scissors-isomorphism $P \rightsquigarrow Q$. Now consider the general case, where instead of a single isometry there is a scissors-isomorphism $g: R \rightsquigarrow S$. This scissorsisomorphism comes with a decomposition

$$
S=\biguplus_{i=1}^{n} S_{i}
$$

By Property A it is possible to decompose each $S_{i}$ into small enough pieces that $P$ will be larger than twice each one; use this to refine $g$ to a scissors-isomorphism such that $P$ is larger than twice $S_{i}$ for all $i$.

Supposing that $P$ is larger than twice $S_{i}$ for all $i$, it is now possible to prove the general case by induction. Write $P^{\prime}=P \uplus \biguplus_{i=1}^{n-1} R_{i}$ and $Q^{\prime}=Q \uplus \biguplus_{i=1}^{n-1} S_{i}$. There is an isometry $g_{n}: R_{n} \rightarrow S_{n}$ and a scissors-isomorphism $P^{\prime} \uplus R_{n} \rightsquigarrow Q^{\prime} \uplus S_{n}$. Since $P$ was larger than twice $S_{n}, P^{\prime}$ will be as well. Thus by the special case above, there is a scissors-isomorphism $P^{\prime} \rightsquigarrow Q^{\prime}$. Repeating this $n-1$ more times produces a scissors-isomorphism $P \rightsquigarrow Q$.

We can summarize the proof of Theorem 1.3 in the following manner: up to precomposition with a scissors-automorphism of $P \uplus R$, any scissors-isomorphism $P \uplus R \rightsquigarrow Q \uplus S$ is the union of a scissors-isomorphism $P \rightsquigarrow Q$ and a scissorsisomorphism $R \rightsquigarrow S$. Therefore, morally speaking, our proof of Theorem 1.3 fails when there are not enough scissors-automorphisms of $P \uplus R$.

Remark. The proof of Theorem 1.3 uses Property A to cut $S$ into pieces that are "small enough". If Property A does not hold, the proof will still work if it is known that if $P \sim Q$, then it is possible to choose $R$ and $S$ "small enough". In fact, when 
Property Z fails for the Grothendieck ring of varieties (see Section 31) it is exactly because this is impossible.

Connections to group homology and $K$-theory. Now that we know that any analysis of the algebra translates directly into an analysis of the geometry, we can work purely in the algebraic setting. Recall that for any group $G$ and any $G$-module $A$, the group $H_{0}(G, A)$ is defined to be the $G$-coinvariants of $A$ :

$$
H_{0}(G, A)=A /(a-g \cdot a \mid a \in A, g \in G) .
$$

The key observation in the connection to scissors congruence is that for any normal subgroup $H$ of $G$,

$$
\mathbb{P}(X, G) \cong H_{0}(G / H, \mathbb{P}(X, H)) .
$$

For example, if we set $H=1$, then $\mathbb{P}(X, G) \cong H_{0}(G, \mathbb{P}(X, 1))$, which tells us that to get from scissors congruence where no motions are allowed to the one where we allow motions from $G$, we quotient out by the action of the group $G$.

This seemingly trivial observation produces a wealth of techniques for analyzing classical scissors congruence. The first large payoff is in the context of translational scissors congruence. Let us restrict ourselves to the case where $X=E^{n}$, and consider only translations as isometries. It is not difficult to construct scissors congruence invariants (called Hadwiger invariants) in this case: for any finite set $\left\{v_{1}, \ldots, v_{k}\right\}$ with $k<n$ of orthogonal vectors, take the invariant

$$
H_{v_{1}, \ldots, v_{k}}: P \longmapsto \sum_{\substack{F \text { face of } \mathrm{P} \\ F \perp v_{i} \\ i=1, \ldots, k}} \operatorname{sgn}(F) \operatorname{Vol}_{n-k}(F) .
$$

Here, the volume is the $(n-k)$-dimensional volume, and the sign of $F$ is determined by how many of the $v_{i}$ 's point to the inside of $P$. For example, if $n=2$ and $k=1$ and $P$ is the unit square, then all such invariants are 0 ; if $k=0$, then it is just the area of the square. On the other hand, if $P$ is a triangle, then for a $v$ which is orthogonal to an edge $e$ of $P$ and is outward-pointing, $H_{v}(P)=\operatorname{length}(e)$. In Sah79, Chapter $3]$, Sah shows that these invariants separate the translational scissors congruence classes. His proof is interesting in part because although he is using very algebraic methods, all of his intuition is geometric. For example, his analysis relies very heavily on canonical decompositions of simplices. Unfortunately, he is unable to determine a complete set of relations between these invariants.

Dupont Dup82, on the other hand, uses a much more algebraic approach by translating the entire problem into the realm of group homology. Dupont constructs a double chain complex whose homology in one direction is $\mathbb{P}\left(E^{n}, \mathbb{R}^{n}\right)$ by definition and whose homology in the other direction is more easily computable. With this he is able to describe the group $\mathbb{P}\left(E^{n}, \mathbb{R}^{n}\right)$ in its entirety in such a way that the relations between Hadwiger invariants become immediately evident: for any set of vectors $\left\{v_{1}, \ldots, v_{k-1}\right\}$ and any subspace $U$ of dimension $n-k$ such that $U \perp\left\{v_{1}, \ldots, v_{k-1}\right\}$,

$$
\sum_{\substack{v \perp U, v \perp v_{i} \\ i=1, \ldots, k-1}} H_{v_{1}, \ldots, v_{k-1}, v}=0 .
$$

Note that this sum always makes sense, as every polytope $P$ only has finitely many nonzero Hadwiger invariants. Dupont's construction is also $O(n)$-equivariant, which means that he can use it to compute $\mathbb{P}\left(E^{n}\right)$ inductively as $H_{0}\left(O(n), \mathbb{P}\left(E^{n}, \mathbb{R}^{n}\right)\right)$. 
These techniques can be pushed further. Dupont and Sah DS82 use them to construct the following exact sequences.

$$
\begin{aligned}
& 0 \longrightarrow \mathbb{R} \stackrel{i}{\longrightarrow} \mathbb{P}\left(E^{n}\right) \stackrel{D}{\longrightarrow} \mathbb{R} \otimes \mathbb{R} / \mathbb{Z} \longrightarrow H_{1}\left(S O(3)^{\delta}, \mathbb{R}^{3}\right) \longrightarrow 0 \\
& 0 \longrightarrow H_{3}\left(S U(2)^{\delta}\right) \longrightarrow \mathbb{P}\left(S^{3}\right) / \mathbb{Z} \stackrel{D}{\longrightarrow} \mathbb{R} \otimes \mathbb{R} / \mathbb{Z} \longrightarrow H_{2}\left(S U(2)^{\delta}\right) \longrightarrow 0 \\
& 0 \longrightarrow\left(K_{3}(\mathbb{C})^{\text {indec }}\right)^{-} \longrightarrow \mathbb{P}\left(\mathcal{H}^{3}\right) \stackrel{D}{\longrightarrow} \mathbb{R} \otimes \mathbb{R} / Z \longrightarrow K_{2} \longrightarrow
\end{aligned}
$$

In the first row, the homomorphism $i$ takes $\ell \in \mathbb{R}$ to the generator represented by a $1 \times 1 \times \ell$ prism. The middle homomorphism $D$ in all three sequences is the Dehn invariant.

The third sequence is of particular interest to us: it is the first occurrence of algebraic $K$-theory in our story. The ${ }^{-}$indicate rationalizing and then taking the -1-eigenspace of the action of complex conjugation. This sequence has been generalized by Goncharov Gon99 to higher dimensions, where he showed that after restriction to those hyperbolic simplices with algebraic vertices (in any model of the hyperbolic plane) the kernel of the generalized Dehn invariant $D$ on $\mathbb{P}\left(\mathcal{H}^{2 n-1}\right)$ is the domain of a homomorphism

$$
\left(\left.\operatorname{ker} D\right|_{\text {alg. }}\right) \otimes \mathbb{Q} \longrightarrow\left(g r_{n}^{\gamma} K_{2 n-1}(\overline{\mathbb{Q}}) \otimes \epsilon(n)\right)^{-} .
$$

Here, $\epsilon(n)$ is $\mathbb{Z}$ with $\mathbb{Z} / 2$ acting on it by $(-1)^{n}$. Goncharov conjectures that in fact these homomorphisms should extend to homomorphisms $\operatorname{ker} D \otimes \mathbb{Q} \rightarrow$ $\left(g r_{n}^{\gamma} K_{2 n-1}(\mathbb{C}) \otimes \epsilon(n)\right)^{-}$.

\section{MiXed-Dimensional SCISSORS CONGRUEnCE}

The preceding two sections discussed cutting up polytopes as though they were physical objects: when a three-dimensional object is cut, the two-dimensional errors are ignored. However, there is no reason to only do scissors congruence from this perspective; it is perfectly reasonable to try and keep track of these intersections as well. In this case, we think of a $k$-simplex as the $k$-dimensional interior of the convex hull of $k+1$ points. A polytope is a finite union of simplices. Then a scissors-isomorphism $P \rightsquigarrow Q$ consists of writing

$$
P=\biguplus_{i=1}^{n} P_{i} \quad \text { and } \quad Q=\biguplus_{i=1}^{n} Q_{i}
$$

with isometries $\varphi_{i}: P_{i} \rightarrow Q_{i}$. In this context we write $\uplus$ to mean that $P_{i} \cap P_{j}=$ $Q_{i} \cap Q_{j}=\emptyset$ for $i \neq j$. This approach to scissors congruence was investigated independently by McMullen [McM89], Morelli [Mor93A], and Goodwillie Goo. In the Euclidean context, the first two authors were working from a purely translational perspective; the third allows all Euclidean transformations.

One advantage of this perspective is that it produces more scissors congruence invariants. When considering all polytopes of dimensions at most $n$, the classical scissors congruence invariants are those that take all polytopes of dimension at most $n-1$ to 0 . When this restriction is discarded, new invariants appear. For example, the Euler characteristic of a polytope $P=\biguplus_{i=1}^{n} P_{i}$ is defined to be

$$
\chi(P)=\sum_{d=0}^{n}(-1)^{d} \#\left\{i \mid \operatorname{dim} P_{i}=d\right\} .
$$


This Euler characteristic is another scissors congruence invariant, but one that is not captured by the purely $n$-dimensional information. What are the scissors congruence invariants in this context?

When $n=0$, the only scissors congruence invariant is cardinality. When $n=1$, there are now two invariants: length and Euler characteristic. These are independent because for any positive $m$ the set of $m$ points has length 0 and Euler characteristic $m$; on the other hand, the half-open interval of length $\ell$ has Euler characteristic 0 . A set with any Euler characteristic and any length can be produced by taking appropriate unions of such polytopes. This is also a complete set of invariants: given any polytope with length $\ell$ and Euler characteristic $g \geq 1$ it is scissors-congruent to the closed segment of length $\ell$ with an extra $g-1$ points added. The case $g=0$ is constructed by taking a half-open interval of length $\ell$. The case $g<0$ is constructed by taking $-g$ open intervals of length $\ell /(-g)$.

When trying to analyze the higher-dimensional scissors congruence invariants, however, the geometric approach becomes prohibitively difficult even in dimension 2. In this case it is necessary to keep track of not only the areas of the polygons but their perimeters and numbers of vertices. We must therefore turn to the algebraic perspective and hope that Property $\mathrm{Z}$ will be satisfied. It is important to notice it is not possible to prove Property $\mathrm{Z}$ by induction on dimension directly: it may be that higher-dimensional polytopes induce relations between lower-dimensional polytopes, since whenever a polytope is dissected, it produces lower-dimensional polytopes at the "cut".

For $G$ a subgroup of the isometry group of $X$, define the scissors congruence groups

$$
\mathcal{E}(X, G)=\left\{\begin{array}{c}
\text { free abelian group on } \\
\text { polytopes in } X
\end{array}\right\} /\left\{\begin{array}{l}
{[P]+[Q]=[P \cup Q]+[P \cap Q],} \\
{[P]=[g \cdot P] \text { for } g \in G .}
\end{array}\right.
$$

The special cases $\mathcal{E}\left(E^{n}, \mathbb{R}^{n}\right)$ and $\mathcal{E}\left(S^{n}, 1\right)$ were originally analyzed by McMullen McM89 and Morelli Mor93C; the special cases $\mathcal{E}\left(E^{n}\right)$ and $\mathcal{E}\left(S^{n}\right)$ were analyzed by Goodwillie Goo. Following the convention for $\mathbb{P}(X)$, write $\mathcal{E}(X)$ for $\mathcal{E}(X, \operatorname{Isom}(X))$. Observe that there is a natural projection $\mathcal{E}(X, G) \rightarrow \mathbb{P}(X, G)$ given by the map

$$
[P] \longmapsto[\overline{\stackrel{\circ}{P}}]
$$

Here, $\bar{P}$ is the closure of the interior of $P$. We define $[\emptyset]=0$.

Theorem 2.1 ([Goo, Theorem 2.5]). If $[P]=[Q]$ in $\mathcal{E}\left(E^{n}, G\right)$, then $P$ and $Q$ are scissors-congruent. In other words, Property $Z$ holds in this context.

This theorem was proved for $\mathcal{E}\left(E^{n}, \mathbb{R}^{n}\right)$ in [McM89, Theorem 4]. However, his proof is not equivariant with respect to the action of $O(n)$ and thus does not generalize to $\mathcal{E}\left(E^{n}, G\right)$.

Proof. It is sufficient to consider the case when $G$ is the entire isometry group of $X$. The general case follows, since if $H \leq G$, then $\mathcal{E}\left(E^{n}, G\right)$ is a quotient of $\mathcal{E}\left(E^{n}, H\right)$. It suffices to construct homomorphisms $\alpha_{k}: \mathcal{E}\left(\mathcal{E}^{\infty}\right) \rightarrow \mathbb{P}\left(E^{k}\right)$ such that the composition

$$
\mathcal{E}\left(E^{k}\right) \longrightarrow \mathcal{E}\left(E^{\infty}\right) \stackrel{\alpha_{k}}{\longrightarrow} \mathbb{P}\left(E^{k}\right)
$$

is the natural projection $\mathcal{E}\left(E^{k}\right) \rightarrow \mathbb{P}\left(E^{k}\right)$. 
Indeed, suppose that such an $\alpha_{k}$ exists for all $k$. The proposition is proved by induction on the dimensions of $P$ and $Q$. If $\operatorname{dim} P=\operatorname{dim} Q=0$, then each is some number of points. Since $\alpha_{0}: \mathbb{P}\left(E^{n}\right) \rightarrow \mathbb{P}\left(E^{0}\right) \cong \mathbb{Z}$ takes each of $P$ and $Q$ to the number of points in it, it follows that $P \cong Q$, as desired.

Now suppose that $\operatorname{dim} P \leq \operatorname{dim} Q=m$. Then $\alpha_{m}[P]=\alpha_{m}[Q]$ in $\mathbb{P}\left(E^{m}\right)$, and thus there exists a decomposition of $P$ and $Q$ in which the dimension- $m$ components match up. Let $P=\biguplus_{i=1}^{k} P_{i}$ and $Q=\biguplus_{i=1}^{k} Q_{i}$ be the decompositions that show that $P$ and $Q$ are classically scissors congruent; thus there may exist $i$ and $j$ such that $P_{i} \cap P_{j} \neq \emptyset$. Let $\stackrel{\circ}{P}_{i}$ be the $m$-dimensional interior of $P_{i}$. Then $\stackrel{\circ}{P}_{i} \cong \stackrel{\circ}{Q}_{i}$; thus if

$$
P^{\prime}=\bigcup_{i=1}^{k}\left(P_{i} \backslash \stackrel{\circ}{P}_{i}\right) \quad \text { and } \quad Q^{\prime}=\bigcup_{i=1}^{k}\left(Q_{i} \backslash \stackrel{\circ}{Q}_{i}\right),
$$

then $\left[P^{\prime}\right]=\left[Q^{\prime}\right]$ in $\mathcal{E}\left(E^{n}\right)$ and $\operatorname{dim} P^{\prime}, \operatorname{dim} Q^{\prime}<m$. By induction this implies that $P^{\prime} \simeq Q^{\prime}$, and this gives us a scissors congruence from $P$ to $Q$, as desired.

It therefore remains to construct $\alpha_{k}$. When $k=0$, define $\alpha_{0}(P)=\chi(P)$, the Euler characteristic. Now suppose that $k>0$. Let $\sigma$ be a $k$-simplex in some triangulation of $P$, and fix any point $u \in \sigma$. Let $\tau_{u}$ be the translation that takes $u$ to the origin. Following Goodwillie [Goo, (11)] define $B_{\sigma}(P)$, the bending of $P$ at $\sigma$ by

$$
B_{\sigma}(P)=\frac{\operatorname{Vol}\left\{x \in S^{n-1} \mid \begin{array}{c}
x \cdot v \leq 0 \text { for all } v \in \tau_{u} P \\
\text { s.t. } v \cdot w=0 \text { for all } w \in \tau_{u} \sigma
\end{array}\right.}{\operatorname{Vol}\left(S^{n-1}\right)} .
$$

The volume here is the $(n-1)$-dimensional volume, and $S^{n-1}$ is the unit sphere in $E^{n}$. The bending at $\sigma$ is independent of the choice of $u$. Define

$$
\alpha_{k}(P)=\sum_{\operatorname{dim} \sigma=k} B_{\sigma}(P)[\bar{\sigma}] .
$$

Here the sum is over any triangulation of $P$ and $[\bar{\sigma}]$ is the image of $\sigma$ inside $\mathbb{P}\left(E^{n}\right)$. When $P$ is a $k$-simplex, take the triangulation with one $k$-simplex, the interior of $P$; therefore the composition of $\alpha_{k}$ with the inclusion of $\mathcal{E}\left(E^{k}\right)$ into $\mathcal{E}\left(E^{n}\right)$ is equal to the projection $\mathcal{E}\left(E^{k}\right) \rightarrow \mathbb{P}\left(E^{k}\right)$.

The bending invariant, as well as its analog $\sigma$ (see [McM89, Theorem 5]) behave a lot like the Dehn invariant in classical scissors congruence: morally speaking, it constructs a comultiplication on the scissors congruence group of polytopes with angles measured in spherical scissors congruence. However, the answer to Open Question 1 is known in the case of the polytope algebra: McM89, Theorem 5] showed that there exists a map $\sigma: \mathcal{E}\left(E^{n}, \mathbb{R}^{n}\right) \rightarrow \mathbb{R} \otimes \bigoplus_{n \geq 0} \mathbb{P}\left(S^{n}, 1\right)$ (analogous to a Hadwiger invariant) which is an injective homomorphism.

Open Question 2. Do Goodwillie's bending invariant and volume separate classes in $\mathcal{E}\left(E^{n}\right)$ ?

Toric varieties and the polytope algebra. The structure of $\mathcal{E}\left(E^{n}, \mathbb{R}^{n}\right)$ is very interesting and turns out to be connected with deep questions about geometry and toric topology. McMullen McM93 used it give a new proof of the $g$-theorem, which gives necessary and sufficient conditions for a sequence of numbers to arise as the numbers of faces of simple polytopes. The sufficiency of these conditions was proved by Billera and Lee [BL81, and necessity was proved by Stanley [Sta80]. However, Stanley's proof relied heavily on algebro-geometric machinery, and a more 
elementary proof was desired. McMullen's proof uses only the deep structure of $\mathcal{E}\left(E^{n}, \mathbb{R}^{n}\right)$ in his proof.

The big advantage of $\mathcal{E}\left(E^{n}, \mathbb{R}^{n}\right)$ over $\mathcal{E}\left(E^{n}\right)$ is that it is an algebra, not just a group. Given two polytopes $P$ and $Q$ in $E^{n}$, define the Minkowski sum of two polytopes to be

$$
P+Q=\{p+q \mid p \in P, q \in Q\}
$$

When $P$ and $Q$ are in orthogonal subspaces $P+Q \cong P \times Q$; otherwise, the sum is more subtle. Define multiplication on $\mathcal{E}\left(E^{n}, \mathbb{R}^{n}\right)$ by $[P][Q]=[P+Q]$. This multiplication is well defined on $\mathcal{E}\left(E^{n}, \mathbb{R}^{n}\right)$ [McM89, Lemma 7]. However, this does not respect scissors congruence classes if rotations are also included in the isometry group, and therefore it is not well defined on $\mathcal{E}\left(E^{n}\right)$. Example:

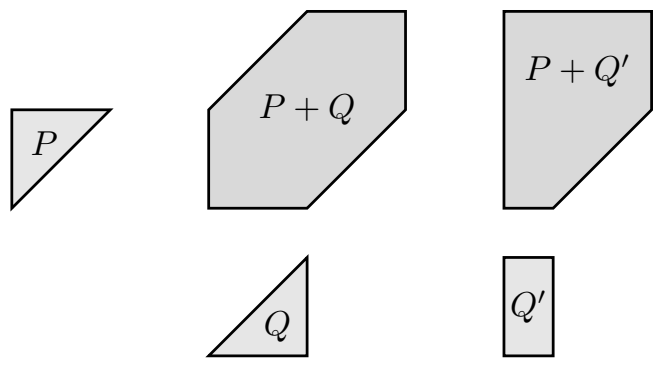

Here, $P$ and $Q$ are isosceles right triangles, and $Q^{\prime}$ is a rectangle with the same area as $Q$ with one side equal to a leg of $Q$. Inside $\mathcal{E}\left(E^{2}\right)[Q]=\left[Q^{\prime}\right]$; however, $[P+Q] \neq\left[P+Q^{\prime}\right]$ as their areas are different.

Dilation by the factor $\lambda$ acts on $\mathcal{E}\left(E^{n}, \mathbb{R}^{n}\right)$, and $\mathcal{E}\left(E^{n}, \mathbb{R}^{n}\right)$ can be written as a sum of weight spaces for this action, with the multiplication on $\mathcal{E}\left(E^{n}, \mathbb{R}^{n}\right)$ respecting this weight space structure. This weight space structure, together with the Minkowski sum multiplication on $\mathcal{E}\left(E^{n}, \mathbb{R}^{n}\right)$, is what gives the polytope structure is power. For more on the polytope algebra and its uses, see McM89.McM93.

We now turn to the connection between $\mathcal{E}\left(E^{n}, \mathbb{R}^{n}\right)$ and toric varieties. A toric variety is one which has a torus (a variety isomorphic to $\left(\mathbb{C}^{\times}\right)^{n}$ ) as a Zariskiopen subset in such a way that the natural action of the torus on itself extends to the whole variety. Toric varieties are particularly interesting because they can be constructed using combinatorial data, and many of their properties (smoothness, properness, etc.) can be deduced purely from the initial data. This makes toric varieties a very nice class of examples to work with. For more on toric varieties, see for example CLS11.

Let $P$ be a polytope in $E^{n}$ whose vertices lie on $\mathbb{Z}^{n}$. A toric variety $X_{P}$ assigned to $P$ can be constructed in the following manner. Consider $\mathbb{Z}^{n}$ as the character lattice of $\left(\mathbb{C}^{*}\right)^{n}$ : a point $\bar{a}=\left(a_{1}, \ldots, a_{n}\right)$ is thought of as the homomorphism $\chi_{\bar{a}}:\left(t_{1}, \ldots, t_{n}\right) \mapsto t_{1}^{a_{1}} \cdots t_{n}^{a_{n}}$. Write $P \cap \mathbb{Z}^{n}=\left\{m_{1}, \ldots, m_{s}\right\}$; this produces a map

$$
\Phi_{P}:\left(\mathbb{C}^{*}\right)^{n} \rightarrow \mathbb{P}^{s} \quad t \mapsto\left(\chi_{m_{1}}(t), \ldots, \chi_{m_{s}}(t)\right)
$$


Define $X_{P}$ to be the Zariski closure of the image of $\Phi_{P} 2$ Many of the properties of $X_{P}$ can be deduced directly from the properties of $P$; for example, $X_{P}$ is smooth if and only if $P$ is smooth in the following sense:

Let $v$ be a vertex of $P$ and $e$ an edge containing $v$. The point $w_{e, v}$ is the lattice point on $e$ closest to $v$. Then $P$ is smooth if for every vertex $v$ the set

$$
\left\{w_{e, v}-v \mid e \text { contains } v\right\}
$$

is a subset of a basis of $\mathbb{Z}^{n}$.

This construction suggests that it should be possible to produce a homomorphism from McMullen's polytope algebra to an algebraic object associated to toric varieties. Fulton and Sturmfels [FS97] construct such a morphism in the following manner. For any rational polytope $P$, take $\Pi(P)$ to be the subalgebra of McMullen's polytope algebra $\Pi$ generated by all polytopes $Q$ such that $P=\lambda Q+R$ for some $\lambda \in \mathbb{Q}$. Let $X_{P}$ be the toric variety associated to $P$. To any ample line bundle $D$ on $X_{P}$ assign a polytope $P_{D}$ (for more detail on this see CLS11, Section 6.2$])$; this polytope will be an element of $\Pi(P)$. Using this observation, Fulton and Sturmfels show that there exists an embedding $\theta: \Pi(P) \hookrightarrow A^{*}\left(X_{P}\right) \otimes \mathbb{Q}$ such that $\theta\left(\left[P_{D}\right]\right)=\exp (D)$ for every ample line bundle $D$ on $X$; for more details see [FS97, Theorem 5.1]. Moreover, the direct $\operatorname{limit}_{\lim } A^{*}(X) \otimes \mathbb{Q}$ over all $n$-dimensional toric varieties $X$ is isomorphic to the entire polytope algebra $\Pi$ [FS97, Theorem 5.2]. Fulton and Sturmfels use this to analyze the structure of toric varieties using the structure of McMullen's polytope algebra. In a later paper Jensen and $\mathrm{Yu}[\mathrm{JY}]$ do a similar analysis for tropical cycles in $\mathbb{R}^{n}$.

The above construction shows how to push information from the geometry of polytopes to the geometry of toric varieties; it is interesting to note that it is also possible to move information in the opposite direction. Given a toric variety $X$, the torus $T$ sitting inside it acts on $X$. Write $K_{T}(X)$ for the $K$-theory group of $T$ equivariant vector bundles on $X$. In Mor93B Morelli constructs a homomorphism $K_{T}(X) \rightarrow \Pi$. He uses this homomorphism to prove [Mor93B, (6)] that there exist coefficients $\mu(F)$, depending only local information about the relatively open face $F$ in $P$, which give an analogous formula to Pick's theorem in arbitrary dimensions:

$$
\text { \#\{lattice points in } P\}=\sum_{F \subseteq P} \operatorname{Vol}_{\operatorname{dim} F}(F) \mu(F) .
$$

Here, the sum is over the faces $F$ of $P$. Pommersheim and Thomas PT04 follow this up with an actual construction of the coefficients $\mu$.

It is currently unknown what the relationship is between Morelli's and FultonSturmfels' homomorphisms.

\section{The Grothendieck Ring of VARIETIES}

Let us now consider a different type of scissors congruence. Fix a base field $k$, and let us consider scissors congruence of varieties over $k$. Here, by a variety we mean a reduced separated scheme of finite type over $k$, or, equivalently, a quasiprojective variety. We can then perform the following "cutting": given a variety $X$

\footnotetext{
${ }^{2}$ For this to work, it is necessary that $P$ is a "very ample" polytope. However, if $P$ is very ample, then so is any integer scaling of it, and any polytope with rational vertices has an integer scaling which is very ample, so for the purposes of this discussion we ignore this detail.
} 
and a closed subvariety $Y$, write

$$
X=Y \uplus(X \backslash Y) .
$$

With this cutting rule, what are scissors congruence invariants of varieties?

If $k$ is finite, then point counting is a scissors congruence invariant: if $X$ has $n$ points over $k$, then each point lies in either $Y$ or $X \backslash Y$, and thus the number of points in $X$ is the number of points in $Y$ plus the number in $X \backslash Y$. However, it is not the case that $X$ is scissors-congruent to a discrete sum of points since the data of a variety contains all points over all extensions of $k$ as well. If $k=\mathbb{C}$, then the Euler characteristic of $X(\mathbb{C})$ can be defined as

$$
\chi_{c}(X)=\sum_{i=0}^{\infty}(-1)^{i} \operatorname{dim}_{\mathbb{Q}} H_{c}^{i}(X(\mathbb{C}) ; \mathbb{Q}) .
$$

Note the use of compactly supported cohomology: this version of the Euler characteristic is not a homotopy invariant, but it acts correctly with respect to cutting. Since $Y$ is a closed subvariety of $X$, the inclusion $Y(\mathbb{C}) \rightarrow X(\mathbb{C})$ gives a long exact sequence in cohomology

$$
\begin{aligned}
\cdots \rightarrow & H_{c}^{i}(X(\mathbb{C}) / Y(\mathbb{C}) ; \mathbb{Q}) \rightarrow H_{c}^{i}(X(\mathbb{C}) ; \mathbb{Q}) \rightarrow H_{c}^{i}(Y(\mathbb{C}) ; \mathbb{Q}) \\
& \rightarrow H_{c}^{i+1}(X(\mathbb{C}) / Y(\mathbb{C}) ; \mathbb{Q}) \rightarrow \cdots .
\end{aligned}
$$

From this it follows that

$$
\begin{aligned}
\chi_{c}(X) & =\sum_{i=0}^{\infty}(-1)^{i} \operatorname{dim}_{\mathbb{Q}} H_{c}^{i}(X(\mathbb{C}) ; \mathbb{Q}) \\
& =\sum_{i=0}^{\infty}(-1)^{i} \operatorname{dim}_{\mathbb{Q}} H_{c}^{i}(Y(\mathbb{C}) ; \mathbb{Q})+\sum_{i=0}^{\infty}(-1)^{i} \operatorname{dim}_{\mathbb{Q}} H_{c}^{i}(X(\mathbb{C}) / Y(\mathbb{C}) ; \mathbb{Q}) \\
& =\chi_{c}(Y)+\sum_{i=0}^{\infty} \operatorname{dim}_{\mathbb{Q}} H_{c}^{i}(X(\mathbb{C}) \backslash Y(\mathbb{C}) ; \mathbb{Q})=\chi_{c}(Y)+\chi_{c}(X \backslash Y) .
\end{aligned}
$$

Thus this Euler characteristic is a scissors congruence invariant. Other scissors congruence invariants include the Hodge characteristic and the motivic zeta function; for more on these see for example [NS11.

Clearly these are not the only scissors congruence invariants of varieties. However, working directly with the geometry becomes very difficult very quickly, and we change to the algebraic analysis. In this case the universal group is called the Grothendieck ring of varieties, as it was first introduced by Grothendieck in a letter to Serre. It is defined to be the free abelian group on varieties over $k$, modulo the relations that for any closed inclusion $Y \rightarrow X$

$$
[X]=[Y]+[X \backslash Y] .
$$

Define a ring structure by $[X]\left[X^{\prime}\right]=\left[X \times X^{\prime}\right]$. This ring is denoted by $K_{0}\left(\mathcal{V}_{k}\right)$; observe that when considered as an abelian group this is the universal additive invariant on varieties over $k$.

Even though $K_{0}\left(\mathcal{V}_{k}\right)$ looks complicated, it is actually possible to do interesting calculations in it. For example, Farb and Wolfson [FW] calculate the class in $K_{0}\left(\mathcal{V}_{k}\right)$ of the variety of rational maps $\mathbb{P}^{1} \rightarrow \mathbb{P}^{m-1}$ of degree $d$ and use this to calculate the number of points over $\mathbb{F}_{q}$ of this variety. Motivic integration is another technique for 
assigning a class in $K_{0}\left(\mathcal{V}_{k}\right)^{3}$ to a subset of the arc space of a variety. This was used by Kontsevich to show that if $X$ and $X^{\prime}$ are smooth complex Calabi-Yau varieties which are birationally equivalent, then they have the same Hodge numbers. (For a short introduction to motivic integration, see Bli11.)

As before, we must determine whether geometric information is lost when considering $K_{0}\left(\mathcal{V}_{k}\right)$. It is known that if $[X]=[Y]$, then $X$ and $Y$ are of the same dimension and are stably birational, and for certain classes of varieties it is known that if $[X]=[Y]$, then $X$ and $Y$ are scissors congruent (see [LS10, Section 5]). The general case of this is very difficult.

Inspired by the previous discussion of scissors congruence, we may wish to restrict our attention to the single-dimensional case: consider the scissors congruences of varieties of dimension $n$ disregarding subvarieties of dimension at most $n-1$. In this case, for any two irreducible varieties $X$ and $X^{\prime},[X]=\left[X^{\prime}\right]$ if and only if $X$ and $X^{\prime}$ are birationally isomorphic. It turns out that here Property $\mathrm{Z}$ holds. To see this, for any variety $X$ write $X=Y \cup \bigcup_{i=1}^{n} X_{i}$, where the $X_{i}$ are the irreducible components dimension $n$ of $X$ and $Y$ has dimension at most $n-1$. Since these intersect only in varieties of dimension at most $n-1$, there exists a Zariski-open subset $\widetilde{X}_{i} \subset X_{i}$ of each $X_{i}$ such that $X=\biguplus_{i=1}^{n} \widetilde{X}_{i} \uplus Y^{\prime}$ where once again $Y^{\prime}$ has dimension at most $n-1$. Therefore

$$
[X]=\sum_{i=1}^{n}\left[\widetilde{X}_{i}\right] .
$$

Thus this group is generated by irreducible varieties. But any scissors congruence between irreducible varieties has exactly one open subset of dimension $n$. Consequently, the single-dimensional case of scissors congruence of varieties is the free abelian group on the birational isomorphism classes of varieties of dimension $n$.

In $K_{0}\left(\mathcal{V}_{k}\right)$, however, Property Z does not hold. Borisov Bor] and Karzhemanov Kar have constructed explicit counterexamples: pairs of varieties $X$ and $Y$ such that $[X]=[Y]$ but $X$ and $Y$ are not scissors congruent. In particular, this implies that when discussing varieties up to scissors congruence, there must exist some kind of geometric invariant which is destroyed by all additive invariants.

Open Question 3. What geometric facts about $X$ and $Y$ does $[X]=[Y]$ imply?

Where is this information lost? The problem lies in adding higher-dimensional varieties. Consider a birational automorphism $\varphi: X \rightarrow X$ of an irreducible variety $X$. This consists of two open subsets $U, V \subseteq X$ and an isomorphism between them. Then

$$
[X \backslash U]=[X]-[U]=[X]-[V]=[X \backslash V]
$$

in $K_{0}\left(\mathcal{V}_{k}\right)$. But it may be (and by the above-mentioned counterexamples, is) the case that $X \backslash U$ and $X \backslash V$ are not necessarily scissors congruent or even birational. Thus "loss" can come from birational automorphisms of varieties which do not extend to scissors-automorphisms of varieties.

Note that this is the same problem that was mentioned in Section 2 In that case it turned out to not be a problem because of the existence of bending; for varieties this does not exist.

\footnotetext{
${ }^{3}$ Actually, $K_{0}\left(\mathcal{V}_{k}\right)\left(\left[\mathbb{A}^{1}\right]^{-1}\right)$; for more on this, see the subsection Connections to motivic integration on page 284
} 
This connection can be expressed more algebraically. Let $K_{0}\left(\mathcal{V}_{k}^{(n)}\right)$ be the free abelian group generated by varieties over $k$ of dimension at most $n$, under the relation that $[X]=[Y]+[X \backslash Y]$ for all closed immersions $Y \hookrightarrow X$. Then there is a homomorphism $K_{0}\left(\mathcal{V}_{k}^{(n-1)}\right) \rightarrow K_{0}\left(\mathcal{V}_{k}^{(n)}\right)$ whose cokernel (by the above discussion) is $\mathbb{Z}\left\{B_{n}\right\}$, where $B_{n}$ is the set of birational isomorphism classes of dimension $n$. Property $\mathrm{Z}$ holds in $K_{0}\left(\mathcal{V}_{k}\right)$ if this homomorphism is injective for all $n$. This is the case when $n \leq 2$ (and in fact the induced homomorphism $K_{0}\left(\mathcal{V}_{k}^{(n)}\right) \rightarrow K_{0}\left(\mathcal{V}_{k}\right)$ is injective when $n \leq 2$; see [LS10]); we also know that this is not the case for some (possibly all) higher $n$ 's.

Connections to motivic integration. Let $X$ be a variety over $k$. The arc space of $X, \mathcal{L}(X)$, is fibered over $X$, with the fiber over a point $x \in X$ consisting of arcs in $X$ centered at $x$. Here, arcs are represented by formal power series. If $X=\operatorname{Spec} R$, then $\mathcal{L}(X)$ will be Spec $R \llbracket t \rrbracket$. It then makes sense to think about the spaces $\mathcal{L}_{n}(X)$, which in the case $X=\operatorname{Spec} R$ will be equal to $\operatorname{Spec} R[t] / t^{n+1}$. (Morally speaking, $\mathcal{L}(X)$ is the space of $\operatorname{arcs}$ in $X$, while $\mathcal{L}_{n}(X)$ is the space of arcs represented by polynomials of degree at most $n$.)

We wish to "measure" subsets of $\mathcal{L}(X)$. As with usual measure theory, this is not always possible, and we must restrict to certain nice subsets. However, the measure should contain more algebraic information than just a number does, and this theory should work over any field. These measures should satisfy certain nice properties: namely, it should be the case that for any two measurable sets $S$ and $T, \mu(S)+\mu(T)=\mu(S \cup T)+\mu(S \cap T)$. This is exactly the defining relation of the Grothendieck ring of varieties, so if we wish to restrict the measure as little as possible, it makes sense to try and define the measure there.

Unfortunately, there is a problem: most subsets that we may wish to measuresuch as for instance the entirety of $\mathcal{L}(X)$-are infinite dimensional. This is not a problem from a scheme-theoretic point of view, but it does raise the issue that the Grothendieck ring of varieties only involves finite-dimensional schemes. Therefore there must be a way to reduce from infinite-dimensional to finite-dimensional schemes.

Consider again the case when $X=\operatorname{Spec} R$. There exist maps

$$
\mathcal{L}(X) \stackrel{\pi_{n}}{\longrightarrow} \mathcal{L}_{n}(X) \stackrel{\pi^{\prime}}{\longrightarrow} X .
$$

Each of these maps is a fibration; the fiber of $\pi_{n}$ is $\mathbb{A}^{\infty}$, and the fiber of $\pi^{\prime}$ is $\mathbb{A}^{n d}$. Fix a point $z \in \mathcal{L}(X)$, and let $y \in\left(\pi^{\prime}\right)^{-1}(z)$. Then the preimages $Y=\pi_{n}^{-1}(y)$ and $Z=\left(\pi^{\prime} \pi_{n}\right)^{-1}(z)$ are isomorphic to $\mathbb{A}^{\infty}$ and $\mathbb{A}^{\infty} \times \mathbb{A}^{n d}$ - and in particular are isomorphic. These are examples of what should be measurable subsets of $X$. However, $Y$ should be much smaller than $Z$ : exactly $\mathbb{A}^{\text {nd }}$ smaller. This is the fundamental observation that makes motivic integration possible: even though most reasonable subsets of $\mathcal{L}(X)$ are infinite-dimensional and therefore do not have nice measures, their codimensions are finite and therefore provide a reasonable basis for comparison.

Let us define this formally. Recall that the ring structure in the Grothendieck ring of varieties is defined by $[X][Y]=[X \times Y]$. Write $\mathbb{L}=\left[\mathbb{A}^{1}\right]$, so that $\left[\mathbb{A}^{k}\right]=\mathbb{L}^{k}$.

Definition 3.1. A subset $A$ of $\mathcal{L}(X)$ is $n$-stable if $\pi_{n}^{-1} \pi_{n}(A)=A$; morally speaking this means that $A \cong \pi_{n}(A) \times \mathbb{A}^{\infty}$. The subset $A$ is called stable if it is $n$-stable for some $n$. 
For a stable set $A$, choose an $n$ such that $A$ is $n$-stable and define

$$
\mu(A)=\left[\pi_{n}(A)\right] \mathbb{L}^{-n d} \in K_{0}\left(\mathcal{V}_{k}\right)\left[\mathbb{L}^{-1}\right] .
$$

The only part of the definition that may fail to be well defined is the choice of $n$ : any $A$ that is $n$-stable is also $(n+1)$-stable. However, in that case there is a fibration $p: \mathcal{L}_{n+1}(X) \rightarrow \mathcal{L}_{n}(X)$, and $\left[p^{-1}(B)\right]=[B] \mathbb{L}^{d}$. (This is true for any fibration: the total space is equal in the Grothendieck ring to the product of the base and the fiber.) Thus for any $(n+1)$-stable $A$, it is the case that

$$
\begin{aligned}
\mu(A) & =\left[\pi_{n+1}(A)\right] \mathbb{L}^{-(n+1) d}=\left[p^{-1} \pi_{n}(A)\right] \mathbb{L}^{-(n+1) d}=\left[\pi_{n}(A)\right] \mathbb{L}^{d} \mathbb{L}^{-(n+1) d} \\
& =\left[\pi_{n}(A)\right] \mathbb{L}^{-n d},
\end{aligned}
$$

as desired.

This measure can be extended to finite disjoint unions of stable sets and then used to define motivic integrals of functions which are nicely constant on stable sets. For more on this perspective, see for example [Bli11, Loo02, DL99.

However, in the spirit of this paper we turn to the question of lost information. In the above paragraphs we blithely inverted $\mathbb{L}$ without worrying that information may be lost. However, Poonen Poo02 showed that $K_{0}\left(\mathcal{V}_{k}\right)$ has zero divisors. It may be the case that $\mathbb{L}$ is a zero divisor and that this localization has killed a large ideal inside the Grothendieck ring of varieties. In fact, it turns out that this is the case: Borisov Bor has constructed varieties $X$ and $Y$ such that $[X] \neq[Y]$ inside the Grothendieck ring of varieties, but $\mathbb{L}[X]=\mathbb{L}[Y]$, so $[X]-[Y]$ is in the kernel of multiplication by $\mathbb{L}$. In fact, amazingly enough, this construction was the same as the one that was mentioned before: it turns out that not only are $X \times \mathbb{A}^{1}$ and $Y \times \mathbb{A}^{1}$ equal in the Grothendieck ring even though $X$ and $Y$ are not, but $X \times \mathbb{A}^{1}$ and $Y \times \mathbb{A}^{1}$ are not birational. This means that they are not scissors congruent, and thus shows that Property $\mathrm{Z}$ does not hold for scissors congruence of varieties.

\section{Algebraic $K$-Theory}

To unify the techniques and ideas of the last three sections, we wish to construct a general theory of scissors congruence which contains the information of the geometric scissors congruence, the algebraic invariants on scissors congruence, and the difference between them. The last section noted that this difference seems to come from scissors-automorphisms of objects. It is interesting to observe that this idea actually appears a lot earlier in the paper, in our discussion of the proof of Zylev's theorem.

In Zylev's theorem, we showed that if $P \uplus R \simeq Q \uplus S$ and $R \simeq S$, then $P \simeq Q$; to construct the explicit scissors-isomorphism $P \rightsquigarrow Q$, we precomposed an arbitrary scissors-isomoprhism $f: P \uplus R \rightsquigarrow Q \uplus S$ with a scissors-automorphism of $P \uplus R$ to get a scissors-isomorphism $f^{\prime}: P \uplus R \rightsquigarrow Q \uplus S$ that restricted to a scissorsisomorphism $P \rightsquigarrow Q$. Now suppose that we are in a situation where Property $\mathrm{Z}$ holds. Then given any scissors-isomorphism $f: P \uplus R \rightsquigarrow Q \uplus S$ and $g: R \rightsquigarrow S$ there exists a scissors-isomorphism $g^{\prime}: P \rightsquigarrow Q$. Thus $f$ differs from a scissorsisomorphism $P \uplus R \rightsquigarrow Q \uplus S$ that restricts to a scissors-isomorphism $P \rightsquigarrow Q$ by the scissors-isomorphism $\left(g \uplus g^{\prime}\right) f^{-1}$. Therefore Property Z holds if and only if there are "enough" scissors-automorphisms to make this always be the case. If we want to be able to measure the failure of Property Z, we therefore need to work in a context where scissors-automorphisms can be analyzed. 
Before explaining how to resolve this question, we digress for motivation for a crash course on algebraic $K$-theory. Those readers comfortable with the $Q$ construction may wish to skip ahead to page 288

Let $R$ be a commutative ring. The algebraic $K$-theory of $R$ studies $R$-modules up to decomposition via exact sequences. Define the group $K_{0}(R)$ to be the free abelian group generated by finitely generated projective $R$-modules $M$, modulo the relations that for every exact sequence

$$
0 \rightarrow M^{\prime} \rightarrow M \rightarrow M^{\prime \prime} \rightarrow 0
$$

there is the relation $[M]=\left[M^{\prime}\right]+\left[M^{\prime \prime}\right]$. As we are primed to see scissors congruence at the moment, we can see that we are doing an algebraic form of scissors congruence of modules. If the given sequence splits, then $M \cong M^{\prime} \oplus M^{\prime \prime}$; using the inspiration of scissors congruence we can say that given such a sequence, then $M$ is scissorscongruent to $M^{\prime} \oplus M^{\prime \prime}$. We can therefore ask if Property Z holds in this context.

Notice that if we only consider finitely generated modules rather than finitely generated projective modules, then Property $\mathrm{Z}$ does not hold: there are $R$-modules which represent equal classes in $K_{0}(R)$ but are not scissors-congruent. For example, if $R=\mathbb{Z}$, then there is an exact sequences

$$
0 \longrightarrow \mathbb{Z} \stackrel{\times 2}{\longrightarrow} \mathbb{Z} \longrightarrow \mathbb{Z} / 2 \longrightarrow 0 .
$$

Thus $[\mathbb{Z}]+[\mathbb{Z} / 2]=[\mathbb{Z}]$ and $[\mathbb{Z} / 2]=0$. However, $\mathbb{Z} / 2$ is clearly not scissors-congruent to the trivial module. When we restrict our attention only to projective $R$-modules, the definition of projectivity exactly rules out this case, and Property $\mathrm{Z}$ holds.

The group $K_{0}(R)$ captures factorization information about the ring $R$. There are many rings that have the same $K_{0}$; for example, whenever $R$ is a field or a PID, $K_{0}(R)=\mathbb{Z}$. Thus if we wish to capture other properties of $R$, other invariants are needed. For example, the group $K_{1}(R)$ is defined to be $G L(R)^{a b}$. Here, $G L(R)$ is the group of infinite matrices which are trivial (equal to the identity) away from a finite submatrix. The determinant homomorphism $G L(R) \rightarrow R^{\times}$factors through $K_{1}(R)$, and we can ask when the homomorphism $K_{1}(R) \rightarrow R^{\times}$is an isomorphism. This is the case when $R$ is a field or a Euclidean domain; however, for general PIDs it is possible for $K_{1}(R) \nRightarrow R^{\times}$(see for example Gra81]). Thus $K_{1}(R)$ can be used to show that a PID is not a Euclidean domain.

In general, the groups $K_{n}(R)$ for $n \geq 0$ are defined as the homotopy groups of a topological space:

$$
K_{n}(R)=\pi_{n} \Omega B Q \operatorname{Proj}(R)
$$

In order to define $B Q \operatorname{Proj}(R)$, we first need to define steps between modules. The goal of the definition is to have a notion of "step" such that passing from a submodule to the whole module is a step, passing from a quotient to the whole module is a step, and the composition of two steps is a step. Define a step from a projective $R$-module $M$ to a projective $R$-module $N$ to be an expression of $M$ as a subquotient of $N$; more formally, it is a submodule $N^{\prime}$ of $N$ and a surjection $N^{\prime} \rightarrow M$. Given a step $\alpha$ from $M$ to $N$ (expressed through a submodule $N^{\prime}$ and a surjection $N^{\prime} \rightarrow M$ ) and a step $\beta$ from $N$ to $P$ (expressed through $P^{\prime}$ and a surjection $P^{\prime} \rightarrow N$ ), compose them to a single step $\beta \alpha$ by letting $P^{\prime \prime} \subseteq P^{\prime}$ be the submodule of $P$ consisting of those elements which land in $N^{\prime}$; in other words, $P^{\prime \prime} \cong N^{\prime} \times_{N} P^{\prime}$. Then there is a surjection $P^{\prime \prime} \rightarrow N^{\prime} \rightarrow M$ and thus a single step from $M$ to $P$. 
Define the pointed CW complex $B Q \operatorname{Proj}(R)$ in the following manner. The 0cells are the finitely generated projective $R$-modules, with basepoint at the trivial module. Attach a 1-cell between $M$ and $N$ for every step from $M$ to $N$. Given a step $\alpha$ from $M$ to $N$ and a step $\beta$ from $N$ to $P$, we think of the 2 -cell as a triangle and attach it in the following manner:

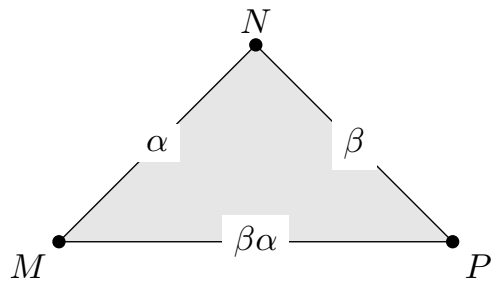

In general, this construction adds an $n$-cell for every $(n+1)$-tuple of projective $R$ modules $M_{0}, \ldots, M_{n}$ and $n$-tuple of steps $\alpha_{i}$ from $M_{i-1}$ to $M_{i}$. Thus $B Q \operatorname{Proj}(R)$ is an infinite-dimensional CW complex. For a more formal explanation of this, see Qui73.

Let us pause to consider why this works, at least for $K_{0}$. By the above definition, $K_{0}(R)=\pi_{1} B Q \operatorname{Proj}(R)$. We do not give a complete proof that this gives an isomorphic group to the original definition, but we give some basic indications as to why this would be the case.

For every module $M$ there exist two steps from 0 to $M$ : the first takes as $M^{\prime}$ the zero module, and the second takes $M^{\prime}=M$, together with the canonical projection $M \rightarrow 0$. These two steps give a loop $[M]$ in $B Q \operatorname{Proj}(R)$, and thus an element of $K_{0}$. Now suppose that there is a short exact sequence

$$
0 \longrightarrow M \longrightarrow N \longrightarrow P \longrightarrow 0 \text {. }
$$

Write $N^{\prime}$ for the image of $M$ inside $N$. Note that there exists a step from $M$ to $N$, given by the submodule $N$ and the trivial surjection $N \rightarrow N$, and a step from $P$ to $N$ given by the submodule $N$ and the surjection $N \rightarrow P$. There is an additional step from 0 to $N$ given by the submodule $N^{\prime}$ and the surjection $N^{\prime} \rightarrow 0$. In $B Q \operatorname{Proj}(R)$ we then have the following picture, where each 0-cell is labeled with its indexing module and each 1-cell is labeled with the submodule representing the step. Note that the 0 node is drawn twice: once at the top and once at the bottom. This is simply for ease in understanding the picture; the reader should imagine that the top and bottom copies are glued together. The shaded regions represent 2-cells.

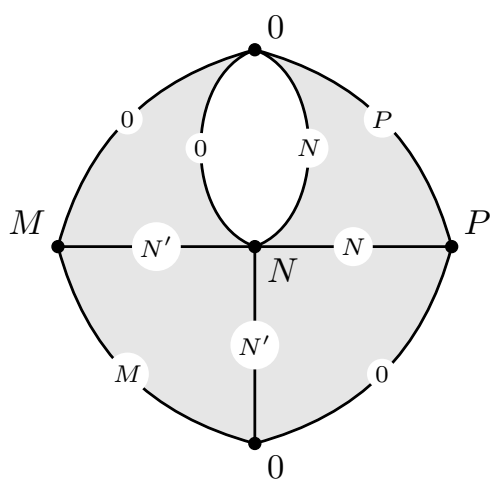


This picture tells us that the path that goes around the entire picture starting at the top and going counter-clockwise, which is represented by $[M]+[P]$, is homotopic to the path which just goes around the unshaded loop in the middle, which is exactly $[N]$. Thus in $\pi_{1} B Q \operatorname{Proj}(R)$ the relation $[N]=[M]+[P]$ holds, as desired inside a model of $K_{0}(R)$.

Summarizing the above construction, the definition of $B Q \operatorname{Proj}(R)$ took the relation that " $M$ is a piece of $N$ " and used it to construct a topological space in such a way that the higher homotopy groups contained information about automorphisms of modules (expressed here through the group $G L(R)$ ).

We now wish to generalize the above construction to produce $K$-groups of scissors congruence problems. In order to consider all of the types of scissors congruence simultaneously, we first construct a general framework for scissors congruence which encapsulates all of the examples considered in Sections 1, 2, and 3. Define an assembler to be a category $\mathcal{C}$, together with a Grothendieck topology: a set $S$ of families of morphisms $\left\{f_{i}: A_{i} \rightarrow A \in \mathcal{C}\right\}_{i \in I}$ for each $A \in$ obC . Such a family encodes the fact that $A$ can be "decomposed" into the pieces $A_{i}$ or, similarly, that the objects $A_{i}$ "cover" $A$. These families must satisfy some axioms which make this geometric relation more precise. Some examples:

(1) Consider the classical scissors congruence of Section 1 Let the objects of $\mathcal{C}$ be polyhedra in $E^{3}$, and define a morphism $\varphi: P \rightarrow Q$ to be an isometry of $E^{3}$ such that $\varphi(P) \subseteq Q$. Thus the morphisms encode the information that $Q$ is "smaller" than $P$. A family $\left\{\varphi_{i}: P_{i} \rightarrow P\right\}_{i \in I}$ is in $S$ if $I$ is finite, the polyhedra $\varphi_{i}\left(P_{i}\right)$ intersect only on the boundaries, and if $\bigcup_{i \in I} \varphi_{i}\left(P_{i}\right)=P$. Thus the families keep track of the fact that $P$ can be decomposed into the $P_{i}$.

(2) Consider the multiple-dimensional scissors congruence of Section 2, Let the objects of $\mathcal{C}$ be open interiors of polytopes of dimension at most $n$ in $E^{n}$. A morphism $\varphi: P \rightarrow Q$ is again an isometry of $E^{n}$ such that $\varphi(P) \subseteq Q$. This time, however, a family $\left\{\varphi_{i}: P_{i} \rightarrow P\right\}_{i \in I}$ is in $S$ if $\varphi_{i}\left(P_{i}\right) \cap \varphi_{j}\left(P_{j}\right)=\emptyset$-i.e. if the images of the polytopes do not intersect at all. Here $I$ is finite and $P=\bigcup_{i \in I} \varphi_{i}\left(P_{i}\right)$. Thus the family keeps track of the fact that $P$ can be decomposed into the $P_{i}$.

(3) Consider the Grothendieck ring of varieties from Section 3 , Let the objects of $\mathcal{C}$ be varieties over $k$, and define a morphism $Y \rightarrow X$ to be a finite composition of open and closed immersions - thus a morphism expresses that $Y$ is isomorphic to a locally closed subset of $X$. To find the families in $S$, we say that $S$ contains all families of the form $\{Y \rightarrow X, X \backslash Y \rightarrow X\}$ where $Y \rightarrow X$ is a closed immersion. The set $S$ must also be closed under refinement: given a family $\left\{f_{i}: A_{i} \rightarrow A\right\}_{i \in I}$ in $S$ and families $\left\{g_{j i}: A_{j i} \rightarrow A_{i}\right\}_{j \in J_{i}}$ for each $i \in I$, the family

$$
\left\{f_{i} g_{j i}: A_{j i} \rightarrow A\right\}_{(i, j) \in \coprod_{i \in I} J_{i}}
$$

must also be in $S$. (Note that in the previous two examples, the set $S$ was also closed under refinement.) Thus this data encodes that varieties can be decomposed by cutting out closed subvarieties finitely many times.

Two objects $A$ and $B$ are scissors-congruent if there exist families $\left\{\varphi_{i}: A_{i} \rightarrow A\right\}_{i \in I}$ and $\left\{\psi_{i}: B_{i} \rightarrow B\right\}_{i \in I}$ and for all $i$ there exist isomorphisms $A_{i} \rightarrow B_{i}$. For more on assemblers see ZakB]. 
Define the scissors congruence group of an assembler $\mathcal{C}$ by

$$
K_{0}(\mathcal{C})=\{\text { free abelian group on objects of } \mathcal{C}\} / \sim
$$

where the relation says that for every family $\left\{f_{i}: A_{i} \rightarrow A\right\}_{i \in I}$ in $S$ there is a relation $[A]=\sum_{i \in I}\left[A_{i}\right]$. For the three examples considered above, $K_{0}$ gives exactly the appropriate scissors congruence group, so this definition makes sense. We wish to define higher $K$-groups $K_{i}(\mathcal{C})$ by constructing a topological space $K(\mathcal{C})$ and taking $K_{i}(\mathcal{C})=\pi_{i} K(\mathcal{C})$. This construction is somewhat more complicated than the construction of $B Q \operatorname{Proj}(R)$, as assemblers lack the duality of projective $R$-modules. Inside $R$-modules there are two ways that a module can be a "piece" of another: it can be a submodule or a quotient, and the construction of $B Q \operatorname{Proj}(R)$ tries to unify these two notions. However, inside an assembler there is no "quotienting" operation: there are only subobjects. This "quotienting" must be put directly into the construction of $K(\mathcal{C})$.

The rest of this section focuses on proving the following theorem:

Theorem 4.2 ([ZakB, Theorem A], ZakC, Theorem A]). Let $\mathcal{C}$ be an assembler. Then the group $K_{0}(\mathcal{C})$ is the free abelian group generated by objects of $\mathcal{C}$, modulo the relation that $[A]=\sum_{i \in I}\left[A_{i}\right]$ for any element $\left\{A_{i} \rightarrow A\right\}_{i \in I}$ of $S$. The group $K_{1}(\mathcal{C})$ is generated by pairs of steps $f_{\epsilon}:\left\{B_{j}\right\}_{j \in J} \rightarrow\left\{A_{i}\right\}_{i \in I}$ for $\epsilon=1,2$.

The outline of the proof is very similar to the construction of $B Q \operatorname{Proj}(R)$ : construct a CW complex which has a loop for every scissors congruence class of objects of $\mathcal{C}$, and whose higher homotopy groups contain scissors automorphism classes of objects. To make the construction easier, the definition of the CW complex only considers decompositions of objects. One of the benefits of considering the homotopy groups is that these formally invert all paths, thus making considering both decompositions and assemblies unnecessary. The actual construction of this space is complicated and requires some extra theory; for the details, see [ZakB Definition 1.11]. We describe the 2-skeleton of the space to give an indication of how the topology works to keep track of both scissors congruence classes and automorphism invariants.

First, as with the construction of $B Q \operatorname{Proj}(R)$ we need to define steps. Observe that $S$ gives a set of relations not between objects of $\mathcal{C}$ but between an object of $\mathcal{C}$ and a tuple of objects of $\mathcal{C}$ : the family $\left\{A_{i} \rightarrow A\right\}_{i \in I}$ expresses the relation that $A$ is equivalent to $\left\{A_{i}\right\}_{i \in I}$. To make this into a relation between objects of the same type, we instead think of $S$ as generating a relation on the set of finite tuples of objects of $\mathcal{C}$. Given two finite tuples $\left\{A_{i}\right\}_{i \in I}$ and $\left\{B_{j}\right\}_{j \in J}$, a "step" between them consists of the following data:

- a map of sets $f: J \rightarrow I$, and

- for each $j \in J$, a morphism $f_{j}: B_{j} \rightarrow A_{f(j)}$

such that for each $i \in I$, the set $\left\{f_{j}: B_{j} \rightarrow A_{i}\right\}_{j \in f^{-1}(i)}$ is in $S$. By an abuse of notation, we write a step as $f:\left\{B_{j}\right\}_{j \in J} \rightarrow\left\{A_{i}\right\}_{i \in I}$. A step is a "formal sum of families in $S$ ", the same way that a tuple of objects is a "formal sum of objects in $\mathcal{C} "$. 
As in (4.1), $K(\mathcal{C})$ is defined to be $\Omega$ of a space which is called $B Q \mathcal{C}$. To construct $B Q \mathcal{C}$, begin with a single 0 -cell. The 1-cells are indexed by steps $f:\left\{B_{j}\right\}_{j \in J} \rightarrow$ $\left\{A_{i}\right\}_{i \in I}$ when $I \cup J \neq \emptyset$. (The trivial step on \{\}$_{\emptyset}$ indexes the 0 -cell.) The data of a 2-cell is a bit more complicated: it encodes both refinement of families in $S$-so decomposing a tuple of objects and then decomposing each of the resulting objects again makes sense - and disjoint union of tuples, which is the group structure on $K_{0}$. (Recall that $K_{0}$ is $\pi_{1}$ of the space that we are constructing.) Each 2-cell is labeled with the following data:

- Finite tuples of objects $\left\{A_{i}\right\}_{i \in I_{\epsilon}},\left\{B_{j}\right\}_{j \in J_{\epsilon}}$, and $\left\{C_{k}\right\}_{k \in K_{\epsilon}}$ for $\epsilon=1,2$.

- Steps $f_{\epsilon}:\left\{B_{j}\right\}_{j \in J_{\epsilon}} \rightarrow\left\{A_{i}\right\}_{i \in I_{\epsilon}}$ and $g_{\epsilon}:\left\{C_{k}\right\}_{k \in K_{\epsilon}} \rightarrow\left\{B_{k}\right\}_{j \in J_{\epsilon}}$.

Such a 2-cell is attached along three 1-cells: first along the 1-cell indexed by the step $g_{2}:\left\{C_{k}\right\}_{k \in K_{2}} \rightarrow\left\{B_{j}\right\}_{j \in J_{2}}$, then along the 1-cell indexed by the step $f_{1}:\left\{B_{j}\right\}_{j \in J_{1}} \rightarrow\left\{A_{i}\right\}_{i \in I_{1}}$, and lastly by the reverse of the cell indexed by the step

$f_{1} g_{1} \amalg f_{2} g_{2}:\left\{C_{k}\right\}_{k \in K_{1} \amalg K_{2}} \rightarrow\left\{A_{i}\right\}_{i \in I_{1} \amalg I_{2}}$.

(This is analogous to the construction described on page 286]) This last step is given by the data of the maps of sets $f_{1} g_{1} \amalg f_{2} g_{2}$ and the morphisms $f_{\epsilon g_{\epsilon}(k)} g_{\epsilon k}: C_{k} \rightarrow$ $A_{f_{\epsilon} g_{\epsilon}(k)}$. Morally speaking, a 2-cell incorporates both "formal addition of steps" and "composition of steps".

Let us "unpack" this definition a little. The group $\pi_{1}$ is generated by the 1-cells of a space, so $\pi_{1}$ of the space we have constructed is generated by the steps between tuples. There is a distinguished family of trivial steps: those steps $\left\{A_{i}\right\}_{i \in I} \rightarrow$ $\left\{A_{i}\right\}_{i \in I}$ where $f=1_{I}$ and $f_{i}=1_{A_{i}}$ for all $i \in I$. This is called the trivial step on $\left\{A_{i}\right\}_{i \in I}$. In order for $\pi_{1}$ to give the desired group, $\pi_{1}$ must be generated by trivial steps. To see this, suppose that $I_{2}=J_{2}=K_{2}=\emptyset$ and $I_{1}=\{*\}$. In this case, there is a cell indexed by a family in $S$ and a refinement of that family (which therefore must also be in $S$ ). The 2 -cell is attached along the step $\left\{f_{j}: B_{j} \rightarrow A\right\}_{j \in J}$ and then the reverse of the step $\left\{f_{g(k)} g_{k}: C_{k} \rightarrow A\right\}_{k \in K}$. In other words, it is expressing the relation that in $\pi_{1}$, a step is equal to any of its refinements. This also holds true for any $I_{1}$, and the generator $f:\left\{B_{j}\right\}_{j \in J} \rightarrow\left\{A_{i}\right\}_{i \in I}$ is equal to the generator given by the trivial step on $\left\{A_{i}\right\}_{i \in I}$. Thus $\pi_{1}$ is indeed generated by trivial steps.

Now consider the case where $I_{1}=J_{1}=K_{1}=\emptyset$ and $g_{2}$ is the trivial step on $\left\{B_{j}\right\}_{j \in J_{2}}$. Then the 2 -cell indexed by this data induces the relation that the generator given by $g_{2}$ is equal to the generator given by $f_{2}$ : thus any step is also equal to the trivial step on its domain. Thus any step $f:\left\{B_{j}\right\}_{j \in J} \rightarrow\left\{A_{i}\right\}_{i \in I}$ is equal to both the trivial step on $\left\{A_{i}\right\}_{i \in I}$ and to the trivial step on $\left\{B_{j}\right\}_{j \in J}$, which exactly gives us the relation $\sim$.

In fact, these two cases together show that the higher homotopy groups of $B Q \mathcal{C}$ contain scissors automorphism information. To see an indication of this, consider any scissors-automorphism of an object $A$ in $\mathcal{C}$. This is a scissors-isomorphism from $A$ to itself, so it consists of two steps $f, g:\left\{B_{j}\right\}_{j \in J} \rightarrow\{A\}$. Consider the following map of a disk $D^{2}$ into $B Q \mathcal{C}$. 


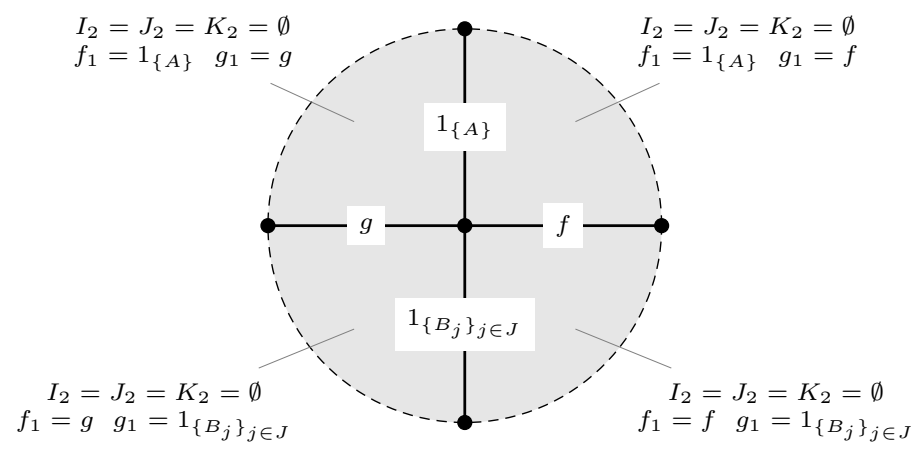

The steps $1_{\left\{A_{i}\right\}_{i \in I}}$ are trivial steps. The ouside dashed circle is represented by the trivial step on \{\}$_{\emptyset}$, so in fact is mapped entirely to the 0 -cell. This is a pointed map of $S^{2}$ into $B Q \mathcal{C}$, and therefore represents an element in $\pi_{2} B Q \mathcal{C}=K_{1}(\mathcal{C})$. Thus any scissors-automorphism of an object in $\mathcal{C}$ (or, more generally, a tuple of objects in $\mathcal{C})$ represents an element of $K_{1}(\mathcal{C})$.

We are not quite done with our discussion of $K_{0}(\mathcal{C})$. We also need it to be the case that the trivial step on the tuple $\left\{A_{i}\right\}_{i \in I}$ is equal to the sum of the trivial steps on the singletons $\left\{A_{i}\right\}$. To see this, consider a 2-cell indexed by data where $f_{\epsilon}$ and $g_{\epsilon}$ are both trivial steps for $\epsilon=1,2$. Then the relation induced on $\pi_{1}$ by this cell is that

$$
\left[\left\{A_{i}\right\}_{i \in I_{1}}\right]+\left[\left\{A_{i}\right\}_{i \in I_{2}}\right]=\left[\left\{A_{i}\right\}_{i \in I_{1} \amalg I_{2}}\right] .
$$

By applying this relation finitely many times, we get exactly the required relation. (Note that this is the only place where finiteness of tuples is used.) Therefore $\pi_{1}$ is generated by trivial steps on singleton tuples, and given a bit more care this proof also shows that it is abelian.

This completes the proof of Theorem 4.2

The big advantage of using $K(\mathcal{C})$ instead of just scissors congruence groups is that this gives an object that can analyze the geometry and the algebra simultaneously. Morally speaking, the geometric data of scissors congruence is in the geometry of the space $K(\mathcal{C})$; the algebraic information is in its homotopy groups. This means that the tools of algebraic topology can be used to study scissors congruence problems in a way that leads to interesting connections. The big disadvantage of $K(\mathcal{C})$ is the same disadvantage that all $K$-theory has: it is incredibly difficult to compute with to get actual answers. Therefore it is not likely that $K$-theoretic techniques can be used to compute scissors congruence groups.

Connections to the Grothendieck ring of varieties. Consider the construction of $K\left(\mathcal{V}_{k}\right)$. The space $B Q \mathcal{V}_{k}$-and thus $K\left(\mathcal{V}_{k}\right)$ - has an increasing filtration by the dimension of the involved varieties, with the filtration degree of a cell being the maximum of the filtration degrees of any variety in the indexing of a cell. The associated graded of this filtration turns out to exactly encapsulate the single-dimensional case discussed above: the $n$-th graded piece of the filtration is constructed out of classifying spaces of birational automorphism groups. More formally,

$$
K\left(\mathcal{V}_{k}\right)^{(n)} / K\left(\mathcal{V}_{k}\right)^{(n-1)} \simeq \bigvee_{[X] \in B_{n}} \Sigma_{+}^{\infty} B \operatorname{Aut}(k(X))
$$

Here, $B_{n}$ is the set of birational isomorphism classes of irreducible varieties of dimension $n$, and $\operatorname{Aut}(k(X))$ is the birational automorphism group of $X$. The symbol 
$\Sigma_{+}^{\infty}$ indicates that we add a disjoint basepoint and then consider this in the category of spectra 4 Note that this is closely analogous to the discussion of the singledimensional case in the scissors congruence of varieties: that discussion produced exactly the $\pi_{0}$-part of this topological space. A filtered space produces a spectral sequence, and this spectral sequence has a beautiful property: the differentials in it give obstructions to Property Z holding!

Unfortunately, birational automorphism groups are incredibly complicated and difficult to work with, and very little is known about their abelianizations. Thus direct computation with this spectral sequence is very difficult. However, it is possible to obtain geometric information out of it even without knowing the exact groups.

Let us consider multiplication by $\mathbb{L}$ in $K_{0}\left(\mathcal{V}_{k}\right)$. Multiplication by $\mathbb{L}$ is a homomorphism $\cdot \mathbb{L}: K_{0}\left(\mathcal{V}_{k}\right) \rightarrow K_{0}\left(\mathcal{V}_{k}\right)$, and Borisov showed that its kernel is nontrivial. In LL03, Theorem 2.3] Larsen and Lunts compute the cokernel of this homomorphism: it is isomorphic (as a ring) to $\mathbb{Z}[S B]$, where $S B$ is the multiplicative monoid of stable birational isomorphism classes of smooth projective varieties. (Recall that two smooth projective varieties $X$ and $Y$ are stably birational if there exist $k$ and $\ell$ such that $X \times \mathbb{P}^{k}$ is birational to $Y \times \mathbb{P}^{\ell}$.)

We now lift the homomorphism $\cdot \mathbb{L}$ to a map $K\left(\mathcal{V}_{k}\right) \rightarrow K\left(\mathcal{V}_{k}\right)$. To do this we construct a map $B Q \mathcal{V}_{k} \rightarrow B Q \mathcal{V}_{k}$, and then apply $\Omega$ to this map. Begin by mapping the 0-cell to the 0-cell. For any 1-cell indexed by a step $f:\left\{Y_{j}\right\}_{j \in J} \rightarrow\left\{X_{i}\right\}_{i \in I}$, map it to the cell indexed by the step $f \cdot \mathbb{L}:\left\{Y_{j} \times \mathbb{A}^{1}\right\}_{j \in J} \rightarrow\left\{X_{i} \times \mathbb{A}^{1}\right\}_{i \in I}$. This step will have the same induced map of sets $J \rightarrow I$ as $f$, and for each $j \in J$, $(f \cdot \mathbb{L})_{j}=f_{j} \times 1_{\mathbb{A}^{1}}$. Similarly, we can multiply all of the indexing data of a 2-cell by $\mathbb{A}^{1}$, and in fact this works on the entirety of $B Q \mathcal{V}_{k}$. Therefore there is a map $\cdot \mathbb{L}: K\left(\mathcal{V}_{k}\right) \rightarrow K\left(\mathcal{V}_{k}\right)$.

Write $C$ for the cofiber of this map so that there is a long exact sequence in homotopy:

$$
K_{1}\left(\mathcal{V}_{k}\right) \stackrel{\cdot \mathbb{L}}{\longrightarrow} K_{1}\left(\mathcal{V}_{k}\right) \longrightarrow \pi_{1} C \longrightarrow K_{0}\left(\mathcal{V}_{k}\right) \stackrel{\cdot \mathbb{L}}{\longrightarrow} K_{0}\left(\mathcal{V}_{k}\right) \longrightarrow \underbrace{\pi_{0} C}_{\cong \mathbb{Z}[S B]} \longrightarrow 0 .
$$

The computation of $\pi_{0} C$ is the Larsen-Lunts result. Since this sequence is exact, the kernel of multiplication by $\mathbb{L}$ is isomorphic to the cokernel of the homomorphism $K_{1}\left(\mathcal{V}_{k}\right) \rightarrow \pi_{1} C$.

The map $\cdot \mathbb{L}$ is compatible with the dimension filtration, and thus $C$ also comes with a filtration by dimension. This gives a map of spectral sequences from the spectral sequence for $K\left(\mathcal{V}_{k}\right)$ to the spectral sequence for $C$. The cokernel of the map on $\pi_{1}$ can be analyzed by considering the graded pieces of it in the spectral sequence. It turns out that elements in the cokernel of the homomorphism $K_{1}\left(\mathcal{V}_{k}\right) \rightarrow \pi_{1} C$ can only arise from nonzero differentials in the spectral sequence for $K_{1}\left(\mathcal{V}_{k}\right)$. By analyzing this structure a bit further, we can conclude the following: any element in the kernel of multiplication by $\mathbb{L}$ represented as $[X]-[Y]$ with $X$ and $Y$ of minimal

\footnotetext{
${ }^{4}$ For those unfamiliar with spectra, one may think of a spectrum as an "abelianization" of a topological space. Spectra correspond to infinite-loop spaces and to homology theories, and all homotopy groups of spectra are abelian groups. For the purposes of the current discussion, the only facts it is important to know are that the spaces $K\left(\mathcal{V}_{k}\right)$ are naturally spectra, and that for a general group $G$ the spectrum $B G$ has many more nonzero homotopy groups than just $\pi_{1}$. In particular, $\pi_{0} \Sigma_{+}^{\infty} B G \cong \mathbb{Z}$ and $\pi_{1} \Sigma_{+}^{\infty} B G \cong G^{a b} \times \mathbb{Z} / 2$; there are also nonzero higher homotopy groups.
} 
dimension will always have $X \times \mathbb{A}^{1}$ and $Y \times \mathbb{A}^{1}$ equal in the Grothendieck ring but not scissors-congruent. Therefore the fact that Borisov's construction worked to find an element in the kernel of multiplication by $\mathbb{L}$ and a counterexample to Property $\mathrm{Z}$ is not a coincidence, but rather an indication of deeper structure in the Grothendieck ring. For more details, see [ZakA].

\section{ABOut THE AUTHOR}

Inna Zakharevich is an L. E. Dickson Instructor at the University of Chicago. Her main research focus is on algebraic $K$-theory with applications to various scissors congruence problems.

\section{REFERENCES}

[BL81] Louis J. Billera and Carl W. Lee, A proof of the sufficiency of McMullen's conditions for $f$-vectors of simplicial convex polytopes, J. Combin. Theory Ser. A 31 (1981), no. 3, 237-255, DOI 10.1016/0097-3165(81)90058-3. MR635368(82m:52006)

[Bli11] Manuel Blickle, A short course on geometric motivic integration, Motivic integration and its interactions with model theory and non-Archimedean geometry. Volume I, London Math. Soc. Lecture Note Ser., vol. 383, Cambridge Univ. Press, Cambridge, 2011, pp. 189-243. MR2885337

[Bor] Lev Borisov. Class of the affine line is a zero divisor in the Grothendieck ring. http://arxiv.org/pdf/1412.6194.

[Car86] Pierre Cartier, Décomposition des polyèdres: le point sur le troisième problème de Hilbert (French), Astérisque 133-134 (1986), 261-288. Seminar Bourbaki, Vol. 1984/85. MR837225 (87i:52010)

[CLS11] David A. Cox, John B. Little, and Henry K. Schenck, Toric varieties, Graduate Studies in Mathematics, vol. 124, American Mathematical Society, Providence, RI, 2011. MR2810322(2012g:14094)

[DL99] Jan Denef and François Loeser, Germs of arcs on singular algebraic varieties and motivic integration, Invent. Math. 135 (1999), no. 1, 201-232, DOI 10.1007/s002220050284. MR.1664700 (99k:14002)

[Dri98] Lou van den Dries, Tame topology and o-minimal structures, London Mathematical Society Lecture Note Series, vol. 248, Cambridge University Press, Cambridge, 1998. MR.1633348(99j:03001)

[DS82] Johan L. Dupont and Chih Han Sah, Scissors congruences. II, J. Pure Appl. Algebra 25 (1982), no. 2, 159-195, DOI 10.1016/0022-4049(82)90035-4. MR662760 (84b:53062b)

[Dup82] Johan L. Dupont, Algebra of polytopes and homology of flag complexes, Osaka J. Math. 19 (1982), no. 3, 599-641. MR676240 (85f:52014)

[Dup01] Johan L. Dupont, Scissors congruences, group homology and characteristic classes, Nankai Tracts in Mathematics, vol. 1, World Scientific Publishing Co., Inc., River Edge, NJ, 2001. MR 1832859 (2002g:52013)

[FS97] William Fulton and Bernd Sturmfels, Intersection theory on toric varieties, Topology 36 (1997), no. 2, 335-353, DOI 10.1016/0040-9383(96)00016-X. MR.1415592 (97h:14070)

[FW] Benson Farb and Jesse Wolfson. Topology and arithmetic of resultants, I: spaces of rational maps. http://arxiv.org/pdf/1506.02713.pdf.

[Gon99] Alexander Goncharov, Volumes of hyperbolic manifolds and mixed Tate motives, J. Amer. Math. Soc. 12 (1999), no. 2, 569-618, DOI 10.1090/S0894-0347-99-00293-3. MR.1649192 (99i:19004)

[Goo] Thomas Goodwillie. Total scissors congruence. http://arxiv.org/abs/1410.7120.

[Gra81] Daniel R. Grayson, $S K_{1}$ of an interesting principal ideal domain, J. Pure Appl. Algebra 20 (1981), no. 2, 157-163, DOI 10.1016/0022-4049(81)90089-X. MR601681 (82m:18005)

[Jes68] Børge Jessen, The algebra of polyhedra and the Dehn-Sydler theorem, Math. Scand. 22 (1968), 241-256 (1969). MR0251633 (40 \#4860) 
[JY] Anders Jensen and Josephine Yu. Stable intersections of tropical varieties. http://arxiv.org/abs/1309.7064.

[Kar] Ilya Karzhemanov. On the cut-and-paste property of algebraic varieties. http://arxiv.org/abs/1411.6084.

[LL03] Michael Larsen and Valery A. Lunts, Motivic measures and stable birational geometry (English, with English and Russian summaries), Mosc. Math. J. 3 (2003), no. 1, 85-95, 259. MR 1996804 (2005a:14026)

[Loo02] Eduard Looijenga, Motivic measures, Astérisque 276 (2002), 267-297. Séminaire Bourbaki, Vol. 1999/2000. MR1886763 (2003k:14010)

[LS10] Qing Liu and Julien Sebag, The Grothendieck ring of varieties and piecewise isomorphisms, Math. Z. 265 (2010), no. 2, 321-342, DOI 10.1007/s00209-009-0518-7. MR2609314 (2011c:14018)

[McM89] Peter McMullen, The polytope algebra, Adv. Math. 78 (1989), no. 1, 76-130, DOI 10.1016/0001-8708(89)90029-7. MR.1021549 (91a:52017)

[McM93] Peter McMullen, On simple polytopes, Invent. Math. 113 (1993), no. 2, 419-444, DOI 10.1007/BF01244313. MR1228132 (94d:52015)

[Mor93A] Robert Morelli, The K-theory of a toric variety, Adv. Math. 100 (1993), no. 2, 154-182, DOI 10.1006/aima.1993.1032. MR 1234308 (94j:14047)

[Mor93B] Robert Morelli, Pick's theorem and the Todd class of a toric variety, Adv. Math. 100 (1993), no. 2, 183-231, DOI 10.1006/aima.1993.1033. MR.1234309 (94j:14048)

[Mor93C] Robert Morelli, A theory of polyhedra, Adv. Math. 97 (1993), no. 1, 1-73, DOI 10.1006/aima.1993.1001. MR 1200289 (94f:52023)

[NS11] Johannes Nicaise and Julien Sebag, The Grothendieck ring of varieties, Motivic integration and its interactions with model theory and non-Archimedean geometry. Volume I, London Math. Soc. Lecture Note Ser., vol. 383, Cambridge Univ. Press, Cambridge, 2011, pp. 145-188. MR2885336

[Poo02] Bjorn Poonen, The Grothendieck ring of varieties is not a domain, Math. Res. Lett. 9 (2002), no. 4, 493-497, DOI 10.4310/MRL.2002.v9.n4.a8. MR.1928868 (2003g:14010)

[PT04] James Pommersheim and Hugh Thomas, Cycles representing the Todd class of a toric variety, J. Amer. Math. Soc. 17 (2004), no. 4, 983-994, DOI 10.1090/S0894-0347-0400460-6. MR2083474 (2005h:14124)

[Qui73] Daniel Quillen, Higher algebraic K-theory. I, Algebraic $K$-theory, I: Higher $K$-theories (Proc. Conf., Battelle Memorial Inst., Seattle, Wash., 1972), Springer, Berlin, 1973, pp. 85-147. Lecture Notes in Math., Vol. 341. MR0338129 (49 \#2895)

[Sah79] C. H. Sah, Hilbert's third problem: scissors congruence, Research Notes in Mathematics, vol. 33, Pitman (Advanced Publishing Program), Boston, Mass.-London, 1979. MR:554756 (81g:51011)

[Sta80] Richard P. Stanley, The number of faces of a simplicial convex polytope, Adv. in Math. 35 (1980), no. 3, 236-238, DOI 10.1016/0001-8708(80)90050-X. MR.563925 (81f:52014)

[Syd65] J.-P. Sydler, Conditions nécessaires et suffisantes pour l'équivalence des polyèdres de l'espace euclidien à trois dimensions (French), Comment. Math. Helv. 40 (1965), 4380. MR0192407 (33 \#632)

[ZakA] Inna Zakharevich. The annihilator of the Lefschetz motive. http://arxiv.org/ abs/1506.06200.

[ZakB] Inna Zakharevich. The K-theory of assemblers. http://arxiv.org/abs/1401.3712.

[ZakC] Inna Zakharevich. On $K_{1}$ of an assembler. http://arxiv.org/abs/1506.06197.

Department of Mathematics, University of Chicago, Chicago, Illinois 